\title{
PERROS PREHISPÁNICOS (CANIS LUPUS FAMILIARIS) DEL PUCARÁ DE TILCARA (PROVINCIA DE JUJUY, ARGENTINA): CONTEXTO CRONOLÓGICO, MORFOLOGÍA Y ROL SOCIAL
}

\author{
PREHISPANIC DOGS (CANIS LUPUS FAMILIARIS) FROM PUCARÁ \\ DE TILCARA SITE (JUJUY PROVINCE, ARGENTINA): \\ CHRONOLOGICAL CONTEXT, MORPHOLOGY AND SOCIAL ROLE
}

\author{
Lucio González Venanzi ${ }^{1,2}$, Francisco Juan Prevosti ${ }^{2,3}$, Carlos Raúl Belotti López de Medina ${ }^{2,4}$, \\ Maximiliano Javier Lezcano ${ }^{2,5}$ y Luciano Prates ${ }^{1,2}$
}

\begin{abstract}
Se presenta la caracterización de restos inéditos de perros (Canis lupus familiaris) del sitio Pucará de Tilcara (Provincia de Jujuy, Argentina), recuperados en dos contextos arqueológicos (Basural 1 y Acrópolis), que representan un NMI $=5$. Los objetivos son definir su cronología, reconstruir su morfología e indagar sobre su estatus social y posibles roles. Para el primer objetivo se realizó el primer fechado-taxón sobre perros en el Noroeste argentino en una muestra del Basural 1, que arrojó una edad de $610 \pm 27$ años ${ }^{14} \mathrm{C}$ AP (1383-1424 años cal. DC) como datación mínima para la presencia de perros prehispánicos en la región. A su vez, el análisis de la evidencia arqueológica de la Acrópolis sugiere su presencia durante la dominación incaica del sitio. La reconstrucción morfológica refuerza el patrón del Noroeste, donde se observa el predominio de perros de tamaño mediano a pequeño. Nuestro análisis sugiere que los perros de la Acrópolis eran animales de compañía y/o prestigio de la élite incaica o de los artesanos especializados, mientras que los ejemplares del Basural 1 fueron utilizados como recursos alimenticios y posiblemente peleteros. Esta es la primera vez que se registra de manera indudable el consumo de perros en el Noroeste argentino prehispánico.

Palabras claves: perros prehispánicos, reconstrucción morfológica, tafonomía, cinofagia, Noroeste argentino.
\end{abstract}

This work presents a detailed description of remains of dogs (Canis lupus familiaris) from the Pucará de Tilcara site (Jujuy Province, Argentina), which were recently recovered from two archaeological contexts $(M N I=5)$. The purpose of this article is to define the chronology and reconstruct the morphology of these dogs, as well as to inquire about their social status and possible roles. To accomplish the first objective, a first taxon-date in northwestern Argentina, carried out on a sample from Midden 1, has established an age of has established an age of $610 \pm 27$ years ${ }^{14} \mathrm{C} \mathrm{BP}(1383-1424$ years cal. $A D)$ as a minimum absolute date for the presence of pre-Hispanic dogs in the region. In turn, the analysis of the archaeological evidence from the second context (Acrópolis) suggests their presence during the Inca domination of the site. Morphological reconstruction lends support to a previously observed pattern, consisting of a predominance of medium to small sized dogs. Our analysis suggests that the Acrópolis dogs could have been pets, as well as prestige goods, of the Inca elite or of specialized craft workers, whereas the specimens from Midden 1 were used as food and possibly fur resources. This is the first time that undeniable evidence of dog consumption among the pre-Hispanic societies of northwestern Argentina has been recorded.

Key words: Pre-Hispanic dogs, morphological reconstruction, taphonomy, cynophagy, Northwestern Argentina.

Los restos de perros prehispánicos (Canis lupus familiaris $=$ Canis familiaris) son poco frecuentes en los sitios arqueológicos de Sudamérica, y la región Andina es la que muestra una mayor abundancia de registros (Prates et al. 2010a; 2010b). Este patrón continental también se observa en Argentina, donde gran parte de los datos proceden del Noroeste (NOA en adelante), aunque en los últimos años también se recuperaron varios ejemplares en el Noreste argentino, Uruguay y sureste de Brasil (Acosta et al.

1 División Arqueología, Museo de La Plata (FCNyM, UNLP), La Plata, Argentina. gonzalezvenanzi@ fcnym.unlp.edu.ar; lprates@fcnym.unlp.edu.ar

2 Consejo Nacional de Investigaciones Científicas y Técnicas (CONICET), Buenos Aires, Argentina.

3 Museo de Ciencias Antropológicas y Naturales, Universidad Nacional de La Rioja (UNLaR). La Rioja, Argentina. protocyon@hotmail.com

4 Instituto de las Culturas (IDECU), Universidad de Buenos Aires. Ciudad Autónoma de Buenos Aires, Argentina. crbelotti@gmail.com

5 Instituto de investigaciones de Diversidad Cultural y Procesos de cambio (IIDYPCA), San Carlos de Bariloche, Argentina. lezcanomaximiliano1@gmail.com 
2011; Castro et al. 2020; López Mazz et al. 2018; Loponte y Acosta 2016; Milheira et al. 2017). En el NOA, los trabajos pioneros de Von Ihering (1913) y Cabrera (1934) propusieron la existencia de perros en tiempos anteriores pero muy cercanos al contacto europeo. La información indica también que en ese momento convivieron distintos morfotipos, con predominio de animales de tamaño pequeño (Belotti López de Medina 2012, 2017; Cabrera 1934; Zetti 1973), y con la presencia de perros sin pelo (Cabrera 1934), característicos de Mesoamérica y de los Andes Centrales (Allen 1920; Schwartz 1997; Valadez Azúa et al. 1998, 2013; Vásquez et al. 2016, 2019). No obstante, el estado de la cuestión presenta deficiencias ostensibles: la escasez de descripciones morfológicas, osteométricas y tafonómicas detalladas y la ausencia de dataciones radiométricas sobre restos de canes domésticos prehispánicos. En consecuencia, tampoco se dispone de información relacionada con los posibles roles cumplidos por este animal en las sociedades precolombinas del área, más allá de su recurrente asociación a la esfera funeraria (Belotti López de Medina 2012; Cabrera 1934; Gallardo 1964-1965; Prates et al. 2010a; Raffino et al. 1977; Zetti 1973). En este trabajo intentaremos evaluar estos planteos acerca de los perros del NOA sobre la base del estudio de registros inéditos procedentes del sitio Pucará de Tilcara (Quebrada de Humahuaca, Jujuy, Argentina).

Nuestros objetivos principales son: (a) avanzar en la definición cronológica de la introducción y presencia prehispánica del perro en el sitio y la región; (b) evaluar el morfotipo de los perros del Pucará de Tilcara y compararlo con otros registros del NOA; (c) determinar si estuvieron presentes perros sin pelo y (d) plantear hipótesis generales sobre el estatus, función o roles de los perros en el contexto social del área. Para definir la cronología se obtuvo el primer fechado-taxón para el NOA y se evaluaron los indicadores cronológicos del contexto de las muestras. Para identificar y definir rasgos fenotípicos se efectuaron análisis morfológicos y morfométrico y para evaluar el estatus y posibles roles de los perros se analizaron las historias tafonómicas de los especímenes.

\section{Materiales y Métodos}

\section{Descripción y contexto arqueológico de las muestras}

Los especímenes de Canis lupus familiaris objeto de este trabajo provienen del sitio Pucará de Tilcara
(Til 1 en adelante). Til 1 se encuentra en el sector medio de la Quebrada de Humahuaca (2335'11.10" S; $65^{\circ} 24^{\prime} 10.40^{\prime}$ W, Figura 1), próximo a la confluencia de los ríos Huasamayo y Grande. Es un asentamiento urbanizado que se extiende sobre un morro de 80 $\mathrm{m}$ de altura, cubriendo una superficie de 17,5 ha (Otero 2013; Zaburlín 2009). Fue poblado a partir del siglo XII DC (Otero et al. 2018), y alcanzaría su máxima extensión hacia el siglo XV DC (Greco y Otero 2016). Til 1 fue identificado como la cabecera de una organización política jerárquica y corporativa (p.ej., jefatura, ayllu, etc.) del periodo de Desarrollos Regionales -ca. 900-1430 años DC- (Tarragó 2000) y como un centro administrativo provincial de primer orden y de producción artesanal especializada (metalurgia, lapidaria, cerámica y tejido) durante el periodo Inca -ca. 1430-1536 años DC- (González 1982; Otero 2013; Williams 2004). Además, existe evidencia de su ocupación hacia el periodo HispanoIndígena (López Geronazzo et al. 2019).

Para este sitio, Schuel (1930:1434) informó el hallazgo de más de 40 cráneos de perros. Dos de ellos fueron estudiados por Cabrera (1934), quien señaló que eran de tamaño pequeño y que posiblemente pertenecieron a los perros sin pelo americanos, conocidos como "perro pila" en el NOA. También Rusconi (2015 [1934]:253) menciona un perro recuperado por Juan Bautista Ambrosetti. Todos estos materiales se encuentran extraviados.

La muestra aquí analizada está conformada por un número mínimo de cinco individuos (NISP = 237; 198 especímenes óseos y 39 dientes). Una parte está depositada en el Museo Etnográfico Juan Bautista Ambrosetti de Buenos Aires (NMI = 2, sobre la base de dos húmeros derechos y dos ulnas izquierdas; NISP = 206; $\mathrm{n}^{\circ}$ de catálogo 8772) y el otro subconjunto fue entregado por Norberto Pelissero a uno de los presentes autores (M.J.L.) (NMI $=3$, sobre la base de tres hemimandíbulas de diferente tamaño, dos derechas y una izquierda; NISP $=31$; con fines analíticos estos especímenes se denominaron Til 1_n1, Til 1_n2, Til 1_n3, Til 1_n4, Til 1_n5, Til 1_n6, Til 1_n7, Til 1_n8, Til 1_n9, Til 1_n10, Til1_n11, Til 1_n12, Til 1_n13, Til 1_n14, Til 1_n15, Til 1_n15, Til1_n16, Til 1_n17, Til1_n18, Til 1_n19, Til 1_n20, Til 1_n21, Til 1_n22, Til 1_n23, Til 1_n24, Til 1_n25, Til 1_n26).

La muestra del Museo Etnográfico J. B. Ambrosetti de Buenos Aires fue recolectada en 1908 por Ambrosetti en la Acrópolis, al norte de la Cima del Poblado (Figura 1), el punto de mayor elevación 


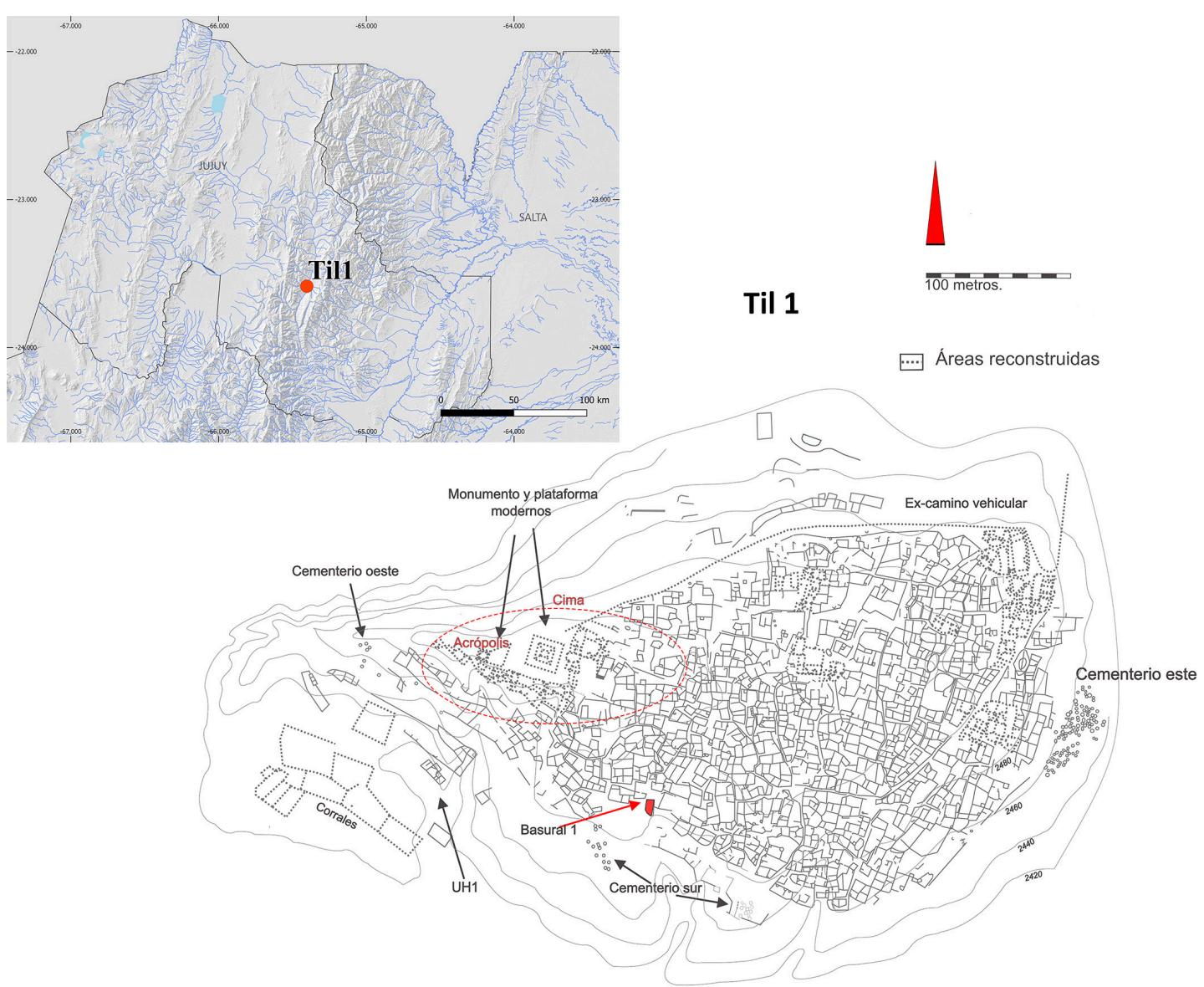

Figura 1. Ubicación de Til 1 y los contextos de hallazgos de Canis lupus familiaris. Modificado de Otero y Tarragó (2017:67). Las fuentes utilizadas para realizar el mapa de la Provincia de Jujuy fueron el Instituto Geográfico Nacional de la República Argentina (modelos de elevación digital y vectores) y Natural Earth (vectores).

Location of Til 1 site and contexts of Canis lupus familiaris findings. Modified from Otero and Tarragó (2017:67). Sources used to make the map of the Jujuy Province were the National Geographic Institute of Argentina (digital elevation models and vectors) and Natural Earth (vectors).

del sitio (Otero 2013). Este sector habría funcionado como un área de viviendas de miembros de la élite incaica (posiblemente líderes religiosos) y también como talleres de artesanos especializados en el trabajo en piedra y metales que fueron auspiciados por el Estado cuzqueño (Otero 2013; Scaro y Otero 2019; Zaburlín y Otero 2014). Sobre la base de la información arqueológica del sitio, sobre todo a partir de evidencia cerámica, se ha planteado que la ocupación humana en la Acrópolis se habría producido principalmente durante la dominación imperial (ca. 1430-1536 años DC) (Otero 2013; Zaburlín y Otero 2014).

La muestra otorgada por Norberto Pelissero fue obtenida en las excavaciones de Casanova en el Basural 1 (Casanova et al. 1976; Pelissero et al. 1997), ubicado en el borde centro-sur de la cúspide de Til 1 (Figura 1) y reexcavado posteriormente (Tarragó y Albeck 1997). El contexto fue interpretado como un área de vivienda y tránsito, que luego fue un sector de descarte. Los fechados radiocarbónicos realizados en la década de 1990 ubicarían el inicio de su utilización en el siglo X DC (Tabla 1) ${ }^{1}$, sin embargo, hoy se supone que Til 1 fue poblado a partir del siglo XII DC (Otero et al. 2018).

\section{Análisis morfológicos, morfométricos, tafonómicos y cuantificación}

La determinación taxonómica y anatómica se realizó mediante la comparación morfológica de los 
Tabla 1. Fechados radiocarbónicos realizados en el Basural 1. Referencias: * corrección por fraccionamiento isotópico de acuerdo al LATyR (Otero 2013); ** edades calibradas con el programa Calib Rev 7.0.4 (Stuiver y Reimer 1993), usando la curva para el hemisferio sur (SHCal13; Hogg et al. 2013).

Radiocarbon datings obtained from the Midden 1. References: * isotopic fractionation correction according to LATyR (Otero 2013); ** ages calibrated with the Calib Rev 7.0.4 program (Stuiver and Reimer 1993), using the curve for the southern hemisphere (SHCal13; Hogg et al. 2013).

\begin{tabular}{cccccc}
\hline Muestra ID & $\begin{array}{c}\text { Técnica } \\
\text { de fechado }\end{array}$ & Muestra & Edad ${ }^{14} \mathrm{C}$ & $\begin{array}{c}\text { Edad calibrada } \\
\text { DC }(2 \sigma)^{* *}\end{array}$ & Fuente \\
\hline D-AMS-033691 & AMS & Óseo (Canis lupus familiaris) & $610 \pm 27$ & $1383-1424$ & Este trabajo \\
\hline LP-546 & Convencional & Carbón & $610 \pm 60^{*}$ & $1298-1441$ & Tarragó y Albeck (1997) \\
\hline LP-544 & Convencional & Carbón & $770 \pm 70^{*}$ & $1184-1393$ & Tarragó y Albeck (1997) \\
\hline LP-485 & Convencional & Carbón & $860 \pm 90^{*}$ & $1028-1313$ & Tarragó y Albeck (1997) \\
\hline LP-486 & Convencional & Carbón & $950 \pm 80^{*}$ & $1013-1270$ & Tarragó y Albeck (1997) \\
\hline LP-531 & Convencional & Carbón & $1020 \pm 80^{*}$ & $955-1220$ & Tarragó y Albeck (1997) \\
\hline LP-466 & Convencional & Carbón & $1160 \pm 80^{*}$ & $763-1046$ & Tarragó y Albeck (1997) \\
\hline
\end{tabular}

especímenes con la colección de Mastozoología del Centro Regional de Investigaciones Científicas y Transferencia Tecnológica de La Rioja (CRILARCONICET) y con bibliografía de referencia (Berta 1989; Evans y De Lahunta 2013; Hildebrand 1954; Lawrence 1967; Loponte y Acosta 2016; Prates et al. 2010b; Tedford et al. 1995). Se hicieron análisis morfométricos según los protocolos de Von Den Driesch (1976) y Morey (1992) para cráneo, Von Den Driesch (1976) para poscráneo y Prevosti (2006) para dientes. Para caracterizar la forma general del cráneo se analizó el índice cefálico (Harcourt 1974). Para estimar la altura a la cruz se emplearon los índices de Harcourt (1974) y Clark (1995) y se la clasificó de acuerdo a los límites propuestos por De Grossi Mazzorin y Tagliacozzo (2000). A su vez, se calculó el índice de la robustez de la diáfisis (Harcourt 1974). Para la estimación etaria se tuvo en cuenta la fusión de las carillas vertebrales y la fusión epifisiaria (Sutton et al. 2018), la erupción dentaria (Sutton et al. 2018) y el grado de desgaste de los dientes en general, y del primer molar inferior en particular (HorardHerbin 2000). Se utilizaron las categorías etarias "juvenil" (menor a 5-7 meses) si el elemento tenía dientes/alvéolos (incisivos, caninos y premolares) correspondientes a la etapa decidua y/o cuando no presentaba las epífisis fusionadas, "subadulto" (aproximadamente entre 5-7 meses y 12-15 meses) si poseía dientes permanentes (incisivos, caninos, premolares y molares) y desgaste leve y/o las epífisis presentaban las líneas de fusión visibles y "adulto" (mayor a 12-15 meses) si el grado de desgaste dentario era moderado/avanzado y/o cuando las epífisis estaban completamente fusionadas.

La determinación sexual de Canis lupus familiaris basada en rasgos cualitativos osteológicos del cráneo presenta diversos inconvenientes (Brassard y Callou 2020), por lo tanto, no se evaluó esta variable. La estimación de la masa corporal se calculó con las fórmulas de regresión del primer molar inferior (Legendre y Roth 1988), del cráneo y la mandíbula (Losey et al. 2015) y del poscráneo (Losey et al. 2017). Los valores obtenidos fueron comparados con otros registros de la especie en el NOA que poseen información morfométrica (Belotti López de Medina 2017; Cabrera 1934; Von Ihering 1913; Zetti 1973).

En el análisis tafonómico se relevó la incidencia de agentes naturales (meteorización, depositación química, marcas de raíces, roedores, carnívoros, fractura en estado seco, pisoteo) y de huellas de origen antrópico (termoalteración, huellas de corte, raspado, impacto, machacado, dientes y fractura en estado fresco) (Binford 1981; Fernández-Jalvo y Andrews 2016; Romero et al. 2016; Saladié et al. 2013). Para interpretar el procesamiento humano de las carcasa se siguieron además los lineamientos basados en carnívoros (Chrószcz et al. 2015; Snyder 1995; Val y Mallye 2011). Se realizó la cuantificación de los especímenes (NISP, NMI) siguiendo la propuesta de Lyman (2008). Los dientes implantados en cráneos y hemimandíbulas se contabilizaron como especímenes (NISP) independientes. 


\section{Resultados}

\section{Individuo 1 (Acrópolis)}

Es un esqueleto de perro casi completo (NISP = 204) (Figura 2) en estado de preservación muy bueno. Si bien no aparece registrado en el diario de campo de Ambrosetti (Zaburlín y Otero 2014), la alta representación anatómica sugiere que se encontraba articulado. En el cráneo (Figura 3) están ausentes varios elementos debido a fracturas producidas en estado seco. Se preservan 10 dientes en el maxilar izquierdo y ocho en el derecho. La serie alveolar está completa en ambos maxilares (I1-M2) y los alvéolos presentan pequeños diastemas entre sí. El índice cefálico $(58,82 \%)$ es compatible con un cráneo mesocéfalo, es decir, con una proporción equilibrada entre su largo (prostion-inion) y ancho (bizigomático). El foramen infraorbitario abre sobre la mitad del P3. El borde anterior de la órbita llega sobre la mitad del P4. El paladar termina a la altura de la porción

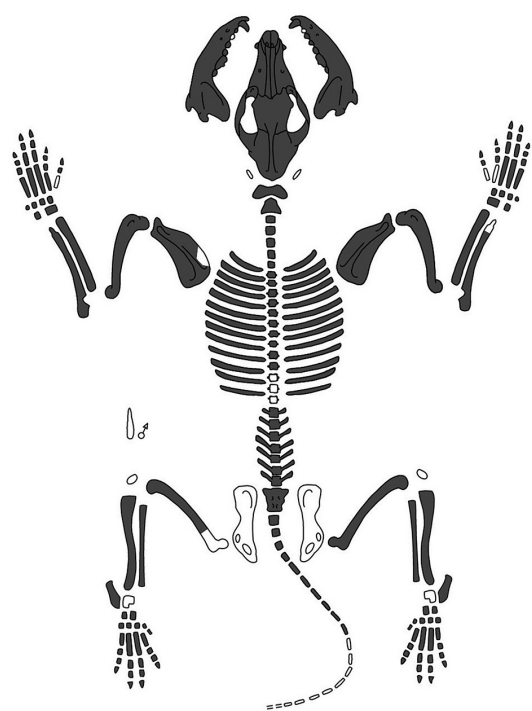

Figura 2. Representación anatómica del individuo 1 recuperado en la Acrópolis de Til 1. Figura muda tomada de ArcheoZoo.org (2013). Anatomical representation of individual 1 recovered from the Acrópolis of Til 1. Blank figure taken from ArcheoZoo.org (2013).

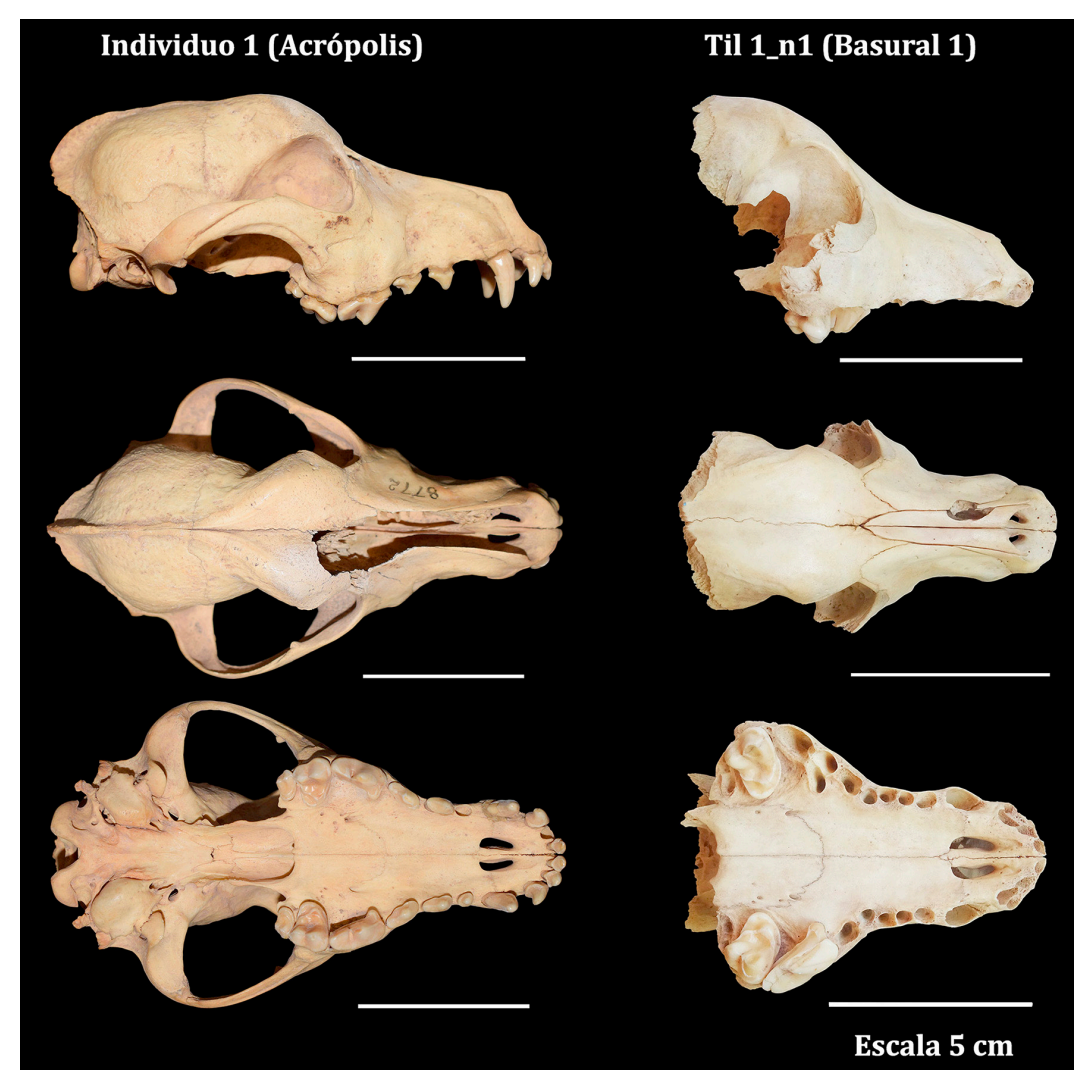

Figura 3. Cráneo del individuo 1 de la Acrópolis y del ejemplar Til 1_n1 del Basural 1 analizados en este trabajo.

Skull of Individual 1 from the Acrópolis and Til 1_n1 specimen of Midden 1 analyzed in this article. 
mesial de los M2; la espina nasal caudal está poco desarrollada, por lo que el borde de las coanas está poco excavado. A nivel del P3, en el rostro, el paladar se angosta significativamente. En vista lateral el rostro es prominente, consecuencia de una frente elevada en relación al hocico. El zigomático es robusto y presenta una muesca marcada para la inserción del músculo masetérico superficial en su cara ventrolateral. Esta muesca se ensancha rostralmente, pero en menor medida que en los cánidos silvestres de América del Sur. El proceso postorbitario es prominente y globoso en vista dorsal. La constricción postorbitaria es angosta, menor al ancho interorbitario. La cresta sagital está bien desarrollada en la parte posterior del cráneo, las líneas temporales se unen justo por detrás del bregma. El inion es aguzado y se proyecta ligeramente por detrás de los cóndilos occipitales en vista lateral. El foramen magnum posee una displasia en su borde dorsal. El basioccipital presenta superficie amplia, las inserciones musculares no están marcadas. Las bullas timpánicas no son grandes y están aplanadas.

Los dientes presentan coronas robustas, bajas y redondeadas. Esto se observa claramente en las cúspides principales de los incisivos, caninos, premolares y M1. Los incisivos superiores son proporcionalmente grandes, en especial el I3, que es caniniforme, y presentan cíngulos linguales muy marcados. El P3 tiene una pequeña cúspide accesoria distal, mientras que el $\mathrm{P} 4$ es robusto, presentando un protocono reducido y un ancho más o menos uniforme en toda su extensión (sin tener en cuenta el protocono). El M1 posee un paracono grande y robusto y un cíngulo labial suave. El grado de desgaste dentario es leve. Las medidas morfométricas de los especímenes craneales se presentan en la Tabla 2 y de los dientes superiores, en la Tabla 3.

Las dos hemimandíbulas están completas (Figura 4). Sus bordes ventrales presentan una curvatura convexa y la serie dentaria en vista oclusal muestra giro helicoidal. Presenta siete dientes implantados en la hemimandíbula izquierda y ocho en la derecha. La serie alveolar está completa en ambos lados: i1-m3. Los premolares presentan un pequeño diastema entre el p3-p4 de ambos lados y entre el p2-p3 del lado izquierdo. El borde posterior de la sínfisis llega aproximadamente hasta la porción distal del p2. El proceso coronoideo es alto y recurvado en sentido caudal, lo cual hace que su borde dorsal sea curvo. El borde anterior de la fosa masetérica comienza a la altura del m3, es amplia, está bien excavada y posee una cresta condilar marcada. Los procesos angulares están bien desarrollados, pero son dorsoventralmente comprimidos y están lingualmente inclinados. Los cóndilos mandibulares se encuentran por encima del nivel del talónido del $\mathrm{m} 1$. Los dientes inferiores presentan el mismo patrón que los superiores, con cúspides bajas y robustas (p.ej., cúspides principales de premolares, canino, trigónido de m1). El p2 derecho no presenta cúspide accesoria distal, mientras en los p3 y p4 sí la poseen; el cíngulo distal está mucho más desarrollado en el p4. El m1 presenta un trigónido grande en relación al talónido, mientras que el metacónido está ausente. El entocónido es considerablemente más pequeño que el hipocónido. El m2 es relativamente corto y posee paracónido bajo y robusto, así como un pequeño hipocónido; el metacónido se encuentra muy reducido y no se observa al entocónido. Las raíces del $\mathrm{m} 2$ izquierdo están fusionadas, mientras que en el derecho están separadas. De acuerdo a las dimensiones del alvéolo del $\mathrm{m} 3$, este diente se encontraba bien desarrollado. La dentición presenta desgaste leve. Las medidas morfométricas de los especímenes hemimandibulares se presentan en la Tabla 4 y de los dientes inferiores, en la Tabla 5.

La masa corporal de este individuo rondaba los $11 \mathrm{~kg}$ (Tabla 6) y la altura a la cruz era de unos $41 \mathrm{~cm}$ ( $\mathrm{n}^{\circ}$ de estimaciones: 26 ; media: 41,1 ; d.e: 0,76 ) (Tabla 7). Sus miembros eran de robustez mediana $\left(\mathrm{n}^{\circ}\right.$ de estimaciones: 7 ; media: 7,38; d.e: 0,54) (Tabla 8). No se detectaron huellas de origen antrópico.

\section{Individuo 2 (Acrópolis)}

Está representado por un húmero y una ulna, ambos con las epífisis fusionadas y estado de preservación muy bueno. Tendría una masa corporal de alrededor de $12 \mathrm{~kg}$ (Tabla 6). A partir del húmero se estableció una altura a la cruz de $45 \mathrm{~cm}$ (Tabla 7) y una robustez mediana (Tabla 8). No se registraron huellas de origen antrópico.

\section{Especímenes del Basural 1}

En esta muestra se identificó un NISP $=31$ (Figura 5), compuesto por especímenes del complejo cráneo-mandíbula (cinco restos óseos y ocho dientes -cinco implantados y tres aislados-) y del poscráneo $($ NISP $=18)$. Se realizó un fechado radiocarbónico (DirectAMS; Washington) que arrojó una edad de 610 27 AP, D-AMS-033691 (hueso, Til 1_n12). El conjunto muestra muy buen estado de preservación. 
Tabla 2. Valores morfométricos ( $\mathrm{mm}$ ) de los restos craneales analizados en este trabajo. Referencias: (i) izquierdo, (d) derecho. Morphometric values ( $\mathrm{mm}$ ) of the cranial remains analyzed in this article. References: (i) left, (d) right.

\begin{tabular}{|c|c|c|c|}
\hline Medida & Individuo 1 & Til 1_n1 & Til 1_n2 \\
\hline \multicolumn{4}{|l|}{ sensu Von Den Driesch (1976) } \\
\hline 1. Largo total: akrokranion-prosthion & 156,74 & - & - \\
\hline 2. Largo condilobasal: borde cóndilos occipitales-prosthion & 146,01 & - & - \\
\hline 3. Largo basal: basion-prosthion & 138,64 & - & - \\
\hline 4. Largo eje basicráneo: basion-synsphenion & 37,96 & - & - \\
\hline 5. Largo eje basifacial: synsphenion-prosthion & 100,08 & - & - \\
\hline 7. Largo neurocráneo: akrokranion-punto medio del frontal & 76,53 & - & - \\
\hline 8. Largo viscerocráneo: nasion-prosthion & ca. 73,01 & 60,38 & - \\
\hline 9. Largo facial: punto medio frontal - prosthion & ca. 92,13 & 75,79 & - \\
\hline 10. Largo máximo de los nasales: nasion - rhinion & ca. 57,89 & 45,06 & - \\
\hline 12. Largo del hocico: borde oral de las órbitas - prosthion & 74,88 & 52,99 & - \\
\hline 13. Largo mediano del paladar: staphylion - prosthion & 77,18 & 68,19 & - \\
\hline 13a. Largo del paladar & 75,67 & - & - \\
\hline 14. Largo de la parte horizontal de los palatinos: staphylion - palatinoorale & 27,16 & 21,99 & - \\
\hline 14a. Largo de la parte horizontal de los palatinos correspondiente a la medida 13a & 25,69 & - & - \\
\hline 15. Largo de la hilera de los dientes molariformes & $55,48(\mathrm{i}) ; 55,14(\mathrm{~d})$ & $46,81(\mathrm{i}) ; 47,31(\mathrm{~d})$ & 53,03 (i) \\
\hline 16. Largo de la hilera de molares & $16,43(\mathrm{i}) ; 16,44(\mathrm{~d})$ & $14,31(\mathrm{i}) ; 14,42(\mathrm{~d})$ & 14,56 (i) \\
\hline 17. Largo de la hilera de premolares & 44,78 (i); 44,24 (d) & 36,15 (i); 36,14 (d) & 42,34 (i) \\
\hline 22. Diámetro mayor de la bulla & 18,7 (i); 17,95 (d) & - & - \\
\hline 23. Ancho mayor de los procesos mastoideos & 55,67 & - & - \\
\hline 24. Ancho dorsal de los meatos auditivos & 54,7 & - & - \\
\hline 25. Ancho mayor de los cóndilos occipitales & 31,23 & - & - \\
\hline 26. Ancho mayor de los procesos paraoccipitales & 55,64 & - & - \\
\hline 27. Ancho mayor del foramen magnum & 17,49 & - & - \\
\hline 28. Altura del foramen magnum: basion-opisthion & 15,61 & - & - \\
\hline 29. Ancho máximo del neurocráneo: euryon-euryon & 49,51 & - & - \\
\hline 30. Ancho bizigomático & 92,21 & - & - \\
\hline 31. Ancho de la constricción postorbitaria & 33,47 & 32,69 & - \\
\hline 32. Ancho del frontal & 44,96 & 36,26 & - \\
\hline 33. Ancho menor entre las órbitas: ectorbitale-ectorbitale & 34,73 & 26,33 & - \\
\hline 34. Ancho mayor del paladar & 56,44 & 54,28 & - \\
\hline 35. Ancho menor del paladar & 29,43 & 27,99 & - \\
\hline 36. Ancho a la altura de los alvéolos de los dientes caninos & 31,69 & 29,64 & - \\
\hline 37. Alto máximo del interior de la órbita & 25,31 (i); 24,86 (d) & $23,48(d)$ & - \\
\hline 38. Altura del cráneo & 49,77 & - & - \\
\hline 39. Altura del cráneo sin la cresta sagital & 47,81 & - & - \\
\hline 40. Altura del triángulo occipital: akrokranion-basion & 38,08 & - & - \\
\hline \multicolumn{4}{|l|}{ sensu Morey (1992) } \\
\hline OI: Largo lateral del rostro: prosthion-anterior de la orbita & 67,63 (i); 66,44 (d) & - & - \\
\hline IM2.Largo de la serie dentaria: prosthion-borde posterior alvéolo M2 & $81,82(\mathrm{i}) ; 82,27$ (d) & - & - \\
\hline
\end{tabular}


Tabla 3. Valores morfométricos ( $\mathrm{mm}$ ) de los dientes superiores analizados en este trabajo. Referencias: (i) izquierdo, (d) derecho.

Morphometric values $(\mathrm{mm})$ of the upper teeth analyzed in this work. References: (i) left, (d) right.

\begin{tabular}{|c|c|c|c|}
\hline Medida & Individuo 1 & Til 1_n1 & Til 1_n6 \\
\hline \multicolumn{4}{|l|}{ sensu Prevosti (2006) } \\
\hline Largo I1 & 4,7 (i); 4,68 (d) & - & - \\
\hline Ancho I1 & 4,68 (i); 4,7 (d) & - & - \\
\hline Largo I2 & 5,01 (i) & - & - \\
\hline Ancho I2 & 5,42 (i) & - & - \\
\hline Largo I3 & 6,98 (i); 7 (d) & - & - \\
\hline Ancho I3 & 5,1 (i); 5,12 (d) & - & - \\
\hline Largo Cl & 8,34 (i); 7,67 (d) & - & $8,16(d)$ \\
\hline Ancho C1 & 4,88 (i); 4,95 (d) & - & $5,3(\mathrm{~d})$ \\
\hline Alto $\mathrm{Cl}$ & 15,3 (i); 14,45 (d) & - & 15,24 (d) \\
\hline Largo P1 & 4,65 (i) & - & - \\
\hline Ancho P1 & 3,75 (i) & - & - \\
\hline Largo P2 & 8,96 (i); 8,33 (d) & - & - \\
\hline Ancho P2 & 4,18 (i); 4,33 (d) & - & - \\
\hline Largo P3 & 10,98 (i); 10,72 (d) & - & - \\
\hline Ancho P3 & 4,98 (i); 4,82 (d) & - & - \\
\hline Largo P4 & 17,26 (i); 17,25 (d) & 16,31 (i) & - \\
\hline Ancho P4 & $9,22(\mathrm{i}) ; 9,16(\mathrm{~d})$ & 9 (i) & - \\
\hline Largo labial M1 & 11,57 (i); 11,55 (d) & 10,83 (i); 10,9 (d) & - \\
\hline Largo lingual M1 & 8,84 (i); 8,07 (d) & 8,53 (i); 8,31 (d) & - \\
\hline Ancho labiolingual M1 & 13,3 (i); 13,34 (d) & 12,64 (i); 12,85 (d) & - \\
\hline Largo M2 & 5,94 (i); 6,3 (d) & - & - \\
\hline Ancho M2 & 7,67 (i); 8,27 (d) & - & - \\
\hline
\end{tabular}

Solo en un fémur y en una hemipelvis se registraron marcas de carnívoros (Figura 6).

Se halló un cráneo pequeño (Til 1_n1) (Figura 3). El foramen infraorbitario se encuentra a la altura de la mitad del P3 y el borde anterior de la órbita está al nivel de la mitad del P4. El paladar es ancho, se angosta de manera abrupta hacia la región anterior y termina a la altura de la porción distal de los M2. El borde ventral de las coanas es prácticamente recto porque la espina nasal caudal está poco desarrollada. Este cráneo presenta una frente pronunciada, con el proceso postorbitario globoso en vista dorsal y con la constricción postorbitaria más ancha que la distancia interorbital. Las suturas no están cerradas. Solo se recuperaron tres dientes ( $\mathrm{P} 4$ izquierdo y ambos M1). La serie alveolar está completa en las dos maxilas (I1-M2) y presentan dos pequeños alvéolos extras por delante de los I3 y tal vez otro, aún más pequeño, delante del I 2 derecho, que corresponderían a los dientes deciduos. No posee diastemas entre los alvéolos, y los alvéolos de los P2-P3 están ligeramente solapados, lo que indica que era un perro de hocico relativamente corto. La dentición presenta desgaste leve. El P4 tiene el protocono pequeño dirigido linguomesialmente, y un suave cíngulo lingual; es robusto y tiene un ancho uniforme si no se tiene en cuenta el protocono. Los M1 están implantados de una forma poco habitual, con la mitad lingual desplazada mesialmente de manera que esta porción se acerca notablemente a la cara lingual del P4. Los M1 muestran un gran paracono, un metacónulo y un hipocono bien desarrollado. El hipocono se conecta con un cíngulo mesial bien desarrollado que rodea al protocono.

Un fragmento de maxilar izquierdo del Basural 1 (Til 1_n2) no posee dientes implantados, presenta siete alvéolos (DC1, C1, P2, P3, P4, M1 y M2) y no tiene fusionada la sutura maxilo-palatina. $\mathrm{Al}$ igual que el cráneo Til 1_n1, tampoco presenta diastemas entre los alvéolos, lo cual sugiere un hocico relativamente corto.

Una hemimandíbula derecha (Til 1_n3) posee borde ventral convexo y giro helicoidal en vista oclusal (Figura 4). El proceso coronoideo es alto y se recurva caudalmente, desarrollando un borde dorsal curvo. El borde anterior de la fosa masetérica comienza inmediatamente por detrás del m3, es amplia, está bien excavada y posee una cresta condilar bien marcada. El proceso angular está bien desarrollado, pero es dorsoventralmente comprimido y está lingualmente inclinado. No posee dientes implantados y falta el alvéolo del p1, sin indicio de haber sido reabsorbido. Tiene un pequeño diastema entre el p2-p3 y otro entre p3-p4. El borde posterior de la sínfisis llega hasta la unión del p2 y el p3. Por lo que puede observarse en los alvéolos del p2 y el m2, sus raíces estaban al menos semifusionadas (los septos alveolares están incompletos). Un fragmento hemimandibular izquierdo (Til 1_n4) presenta los alvéolos c1,p2,p3, p4 y porción mesial del $\mathrm{m} 1$; no tiene diastemas entre los alvéolos (Figura 4). La hemimandíbula Til 1_n5 es pequeña; presenta el borde ventral ligeramente convexo, giro helicoidal de la serie dentaria en vista oclusal y el proceso coronoideo está recurvado caudalmente (Figura 4). Dentro de la cavidad alveolar, 


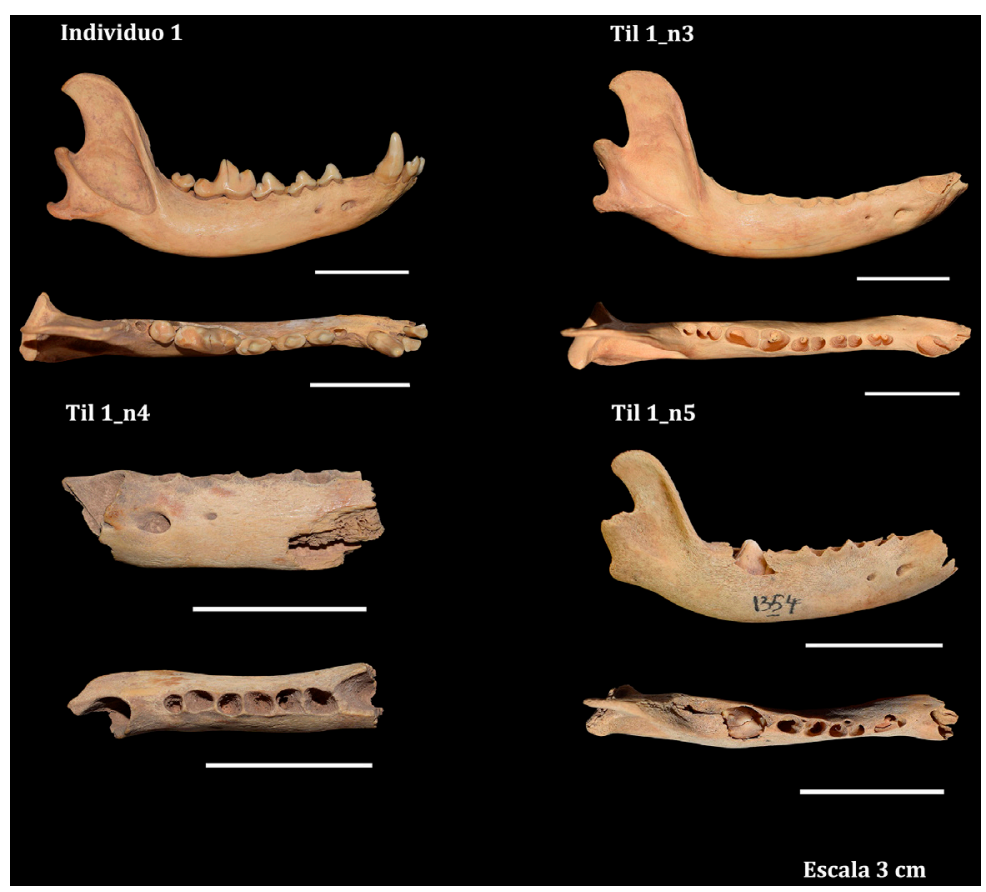

Figura 4. Hemimandíbulas del Individuo 1 de la Acrópolis y Til 1_3, Til 1_n4 y Til 1_n5 del Basural 1 analizadas en este trabajo.

Hemimandibles of Individual 1 from the Acrópolis and Til 1_3, Til 1_n4, and Til 1_n5 of Midden 1 analyzed in this article.

Tabla 4. Valores morfométricos (mm) de los restos hemimandibulares analizados en este trabajo. Referencias: (i) izquierdo, (d) derecho. Morphometric values ( $\mathrm{mm}$ ) of the hemimandible remains analyzed in this work. References: (i) left, (d) right.

\begin{tabular}{|c|c|c|c|c|}
\hline Medida & Individuo 1 & Til 1_n3 & Til 1_n4 & Til 1_n5 \\
\hline \multicolumn{5}{|l|}{ sensu Von Den Driesch (1976) } \\
\hline 1. Largo total: proc. cóndilo-infradentale & 114,71 (i); $114,82(\mathrm{~d})$ & $118,38(d)$ & - & - \\
\hline 2. Largo: proc. angular-infradentale & 116,79 (i); $117,02(d)$ & $118,64(\mathrm{~d})$ & - & - \\
\hline 3. Largo: hendidura entre el proc. cóndilo y proc. angular-infradentale & $110,67(\mathrm{i}) ; 110,71(\mathrm{~d})$ & $113,65(\mathrm{~d})$ & - & - \\
\hline 4. Largo: proc. cóndilo-borde distal alvéolo c1 & 99,61 (i); $99,43(\mathrm{~d})$ & $100,48(d)$ & - & - \\
\hline 5. Largo: hendidura entre el proc. cóndilo y proc. angular-borde distal alvéolo c1 & 95,15 (i); $95,71(\mathrm{~d})$ & $97,16(d)$ & - & $66,18(\mathrm{~d})$ \\
\hline 6. Largo: proc. angular- borde distal alvéolo c1 & 101,69 (i); 102,03 (d) & $102,42(d)$ & - & $66,96(d)$ \\
\hline 7. Largo: borde distal alvéolo m3- borde distal alvéolo c1 & 67,55 (i); 67,27 (d) & $68,88(\mathrm{~d})$ & - & - \\
\hline 8. Largo entre los alvéolos m3-p1 & 61,84 (i); 61,7 (d) & - & - & - \\
\hline 9. Largo entre los alvéolos m3-p2 & 57,84 (i); 57,83 (d) & $62,34(d)$ & - & - \\
\hline 10. Largo entre los alvéolos m3-m1 & 30,44 (i); $31,33(\mathrm{~d})$ & $32,44(d)$ & - & - \\
\hline 11. Largo entre los alvéolos p4-p1 & 33,33 (i); 32,19 (d) & - & - & - \\
\hline 12. Largo entre los alvéolos p4-p2 & 28,96 (i); 28,57 (d) & $31,01(\mathrm{~d})$ & 29,85 (i) & - \\
\hline 14. Largo del alvéolo carnicero & 16,96 (i); 17,66 (d) & $19,66(d)$ & - & - \\
\hline 17. Máximo grosor del cuerpo mandibular & $9,65(\mathrm{i}) ; 9,94(\mathrm{~d})$ & $11,15(\mathrm{~d})$ & - & $9,12(d)$ \\
\hline 18. Alto de la rama vertical & 46,95 (i); 46,54 (d) & $46,88(\mathrm{~d})$ & - & $28,15(\mathrm{~d})$ \\
\hline 19. Alto de la mandíbula detrás del m1 & 21,12 (i); 20,85 (d) & $21,26(d)$ & - & $15,59(\mathrm{~d})$ \\
\hline 20. Alto de la mandíbula entre p2-p3 & 16,34 (i); 15,93 (d) & $16,88(\mathrm{~d})$ & 15,84 (i) & $14,46(d)$ \\
\hline
\end{tabular}


Tabla 5. Valores morfométricos ( $\mathrm{mm})$ de los dientes inferiores analizados en este trabajo.

Referencias: (i) izquierdo, (d) derecho.

Morphometric values ( $\mathrm{mm}$ ) of the lower teeth analyzed in this work. References: (i) left, (d) right.

\begin{tabular}{|c|c|c|c|}
\hline Medida & Individuo 1 & Til 1_n7 & Til 1_n8 \\
\hline \multicolumn{4}{|l|}{ sensu Prevosti (2006) } \\
\hline Largo i2 & 4,44 (d) & - & - \\
\hline Ancho i2 & $4,11(\mathrm{~d})$ & - & - \\
\hline Largo i3 & 4,57 (i); 4,89 (d) & - & - \\
\hline Ancho i3 & 4,75 (i); 4,52 (d) & - & - \\
\hline Largo c1 & 8,99 (i); 8,48 (d) & 8,57 (i) & - \\
\hline Ancho c1 & $5,8(\mathrm{i}) ; 5,78(\mathrm{~d})$ & 5,48 (i) & - \\
\hline Alto c1 & 14,12 (i); 13,84 (d) & 14,39 (i) & - \\
\hline Largo p1 & 4,12 (i) & - & - \\
\hline Ancho p1 & 3,1 (i) & - & - \\
\hline Largo p2 & 7,77 (d) & - & - \\
\hline Ancho p2 & 4,22 (d) & - & - \\
\hline Largo p3 & 9,73 (i); 9,47 (d) & - & - \\
\hline Ancho p3 & 4,67 (i); 4,45 (d) & - & - \\
\hline Largo p4 & 10,84 (i); 10,65 (d) & - & - \\
\hline Ancho p4 & 5,47 (i); $5,21(\mathrm{~d})$ & - & - \\
\hline Largo m1 & 20,07 (i); 19,93 (d) & - & $19,22(d)$ \\
\hline Largo trigónido m1 & 13,15 (i); 12,84 (d) & - & $13,21(d)$ \\
\hline Ancho trigónido m1 & 7,75 (i); 7,83 (d) & - & $8,06(d)$ \\
\hline Ancho talónido m1 & 7,29 (i); 6,83 (d) & - & $7,55(d)$ \\
\hline Largo $\mathrm{m}^{2}$ & 7,6 (i); 7,84 (d) & - & - \\
\hline Ancho $\mathrm{m}^{2}$ & $6,29(\mathrm{i}) ; 6,18(\mathrm{~d})$ & - & - \\
\hline
\end{tabular}

sin erupcionar, se encuentran el p2 y el m1; además, presenta los alvéolos correspondientes a los dientes deciduos. El m1 muestra un metacónido pequeño, dividido por un surco.

El esqueleto poscraneal es el más abundante del conjunto (Figura 5), está representado por elementos del esqueleto axial (2) y, principalmente, del esqueleto apendicular (16), correspondientes a juveniles, subadultos y adultos (Tabla 6). El cálculo de altura a la cruz se pudo realizar en un metacarpo $\mathrm{V}$ y un fémur (Tabla 7); este último presentó una robustez mediana (Tabla 8).

La evidencia de manipulación antrópica en los especímenes del Basural 1 es abundante (NISP $=15$;
Tabla 9), principalmente huellas de corte (Figuras 6 y 7). No se detectaron elementos con signos de termoalteración.

\section{Discusión}

\section{Cronología}

La cronología de Canis lupus familiaris para sitios arqueológicos del NOA no ha sido definida con precisión porque no se dispone de fechados-taxón y porque los contextos de hallazgos en general no están bien documentados (p.ej., Cabrera 1934; Gallardo 1964 1965; Von Ihering 1913). La información disponible indica su presencia durante el periodo de Desarrollos Regionales, ca. 900-1430 años DC (Belotti López de Medina 2012, 2017; Raffino et al. 1977; Zetti 1973) y periodo Inca, ca. 1430-1536 años DC (Rodríguez Loredo 1997-1998). Los datos arqueológicos generados para el sector de la Cima de Til 1 (Otero 2013; Zaburlín y Otero 2014), donde se emplaza la Acrópolis, sugieren que los individuos 1 y 2 corresponden al periodo de dominación incaica del sitio. De todas formas, teniendo en cuenta la cerámica encontrada por Ambrosetti en la Acrópolis, por ejemplo, un puco Humahuaca Negro sobre Rojo y otro Interior Negro Pulido (Otero 2013), no puede descartarse una antigüedad preincaica tardía, o que hayan correspondido a inicios del periodo Hispano-Indígena, como indican nuevas dataciones en la Cima (López Geronazzo et al. 2019). El fechadotaxón realizado sobre el espécimen del Basural 1 es el primero para la región y cae en tiempos prehispánicos (1383-1424 años cal. DC), dentro del periodo de Desarrollos Regionales II o Tardío (ca. 1250-1430 años DC) de acuerdo a la periodización de la Quebrada de Humahuaca (Nielsen 2001, 2007), hacia finales del uso del montículo (Tabla 1).

\section{Morfología}

La reconstrucción morfológica indica que los perros de Til 1 eran de tamaño mediano pequeño a pequeño. La comparación de la masa corporal con otros registros arqueológicos de la especie en el NOA (Figura 8) muestra el predominio de este tipo de perros (menores a $15 \mathrm{~kg}$ ), principalmente, en la Quebrada de Humahuaca (Belotti López de Medina 2017; Cabrera 1934) y en la Quebrada del Toro (Zetti 1973). El único cráneo que pudo asignarse tipológicamente (individuo 1) corresponde a un mesocéfalo (posición intermedia entre el "Grupo N" y el "Grupo A" de 
Tabla 6. Estimaciones de la edad y masa corporal (kg) para los restos de Canis lupus familiaris recuperados en Til 1. Age and body mass estimates $(\mathrm{kg})$ for the Canis lupus familiaris remains recovered in Til 1 site.

\begin{tabular}{|c|c|c|c|c|c|c|}
\hline Contexto arqueológico & Individuo/ Espécimen & Edad & $\begin{array}{c}\mathrm{N}^{\circ} \\
\text { estimaciones }\end{array}$ & Media (kg) & Mín. & Máx. \\
\hline \multirow{2}{*}{ Acrópolis } & Individuo 1 & Subadulto & 121 & 11,23 & 6,27 & 8,33 \\
\hline & Individuo 2 & Adulto & 8 & 12,4 & 9,67 & 16,95 \\
\hline \multirow{26}{*}{ Basural 1} & Cráneo (Til 1_n1) & Juvenil/subadulto & 13 & 9,05 & 5,13 & 15,25 \\
\hline & Maxilar (Til 1_n2) & Juvenil/subadulto & 3 & 12,8 & 9,87 & 14,76 \\
\hline & Hemimand. (Til 1_n3) & Subadulto/adulto & 15 & 13,74 & 10,13 & 19 \\
\hline & Hemimand. (Til 1_n4) & Subadulto/adulto & 2 & 10,21 & 8,3 & 12,11 \\
\hline & Hemimand. (Til 1_n5) & Juvenil & 6 & 5,58 & 3,83 & 8,39 \\
\hline & C1 (Til 1_n6) & Subadulto/adulto & - & - & - & - \\
\hline & c1 (Til 1_n7) & Subadulto/adulto & - & - & - & - \\
\hline & m1 (Til 1_n8) & Subadulto/adulto & 1 & 13,2 & - & - \\
\hline & Escápula (Til 1_n9) & Subadulto/adulto & 2 & 8,25 & 7,56 & 8,94 \\
\hline & Escápula (Til 1_n10) & Subadulto/adulto & 2 & 8,53 & 7,7 & 9,37 \\
\hline & Húmero (Til 1_n11) & Adulto & - & - & - & - \\
\hline & Húmero (Til 1_n12) & Adulto & 1 & 7,63 & - & - \\
\hline & Ulna (Til 1_n13) & Adulto & 3 & 10,5 & 7,66 & 14,45 \\
\hline & Fémur (Til 1_n14) & Subadulto & 1 & 7,21 & - & - \\
\hline & Escápula (Til 1_n15) & Adulto & 4 & 10,21 & 8,82 & 11,41 \\
\hline & Húmero (Til 1_n16) & Subadulto/adulto & - & - & - & - \\
\hline & Húmero (Til 1_n17) & Subadulto/adulto & - & - & - & - \\
\hline & Ulna (Til 1_n18) & Adulto & 3 & 11,47 & 9,08 & 14,51 \\
\hline & Hemipelvis (Til 1_n19) & Adulto & - & - & - & - \\
\hline & Hemipelvis (Til 1_n20) & Adulto & - & - & - & - \\
\hline & Fémur (Til 1_n21) & Adulto & 5 & 11,32 & 10,54 & 12,38 \\
\hline & Radio (Til 1_n22) & Juvenil & 4 & 5,58 & 3,79 & 7,66 \\
\hline & Vértebra toráxica (Til 1_n23) & Juvenil & - & - & - & - \\
\hline & Radio (Til 1_n24) & Adulto & - & - & - & - \\
\hline & Metacarpo V (Til 1_n25) & Adulto & - & - & - & - \\
\hline & Axis (Til 1_n26) & Adulto & - & - & - & - \\
\hline
\end{tabular}

Brothwell et al. 1979:Figura 7). A este grupo también podría sumarse la hemimandíbula Til 1_n3, a juzgar por la presencia de diastemas entre sus alvéolos. Teniendo en cuenta el apiñamiento alveolar, es posible que un cráneo (Til 1_n1), un maxilar (Til 1_n2) y una hemimandíbula (Til 1_n4) fuesen perros con hocicos relativamente cortos. Este morfotipo fue registrado por Zetti (1973) en el sitio Tastil (Quebrada del Toro) y, a nivel macrorregional, por ejemplo, por Brothwell et al. (1979:Figura 7, “Grupo N”) en Perú. Por otra parte, el cráneo Til 1_n1 presenta una similitud notable con el ejemplar de la Tumba 6 de La Isla de Tilcara (2624), tanto morfológica como morfométricamente (Belotti López de Medina 2012); la proximidad temporal y geográfica de los contextos sugiere que estos perros pudieron pertenecer a una misma población. Los 
Tabla 7. Estimaciones de la altura a la cruz $(\mathrm{cm})$ para Canis lupus familiaris de Til 1. Referencias: (i) izquierdo, (d) derecho.

Height estimates at the withers (cm) for Canis lupus familiaris from the Til 1 site. References: (i) left, (d) right.

\begin{tabular}{lccc}
\hline Elemento & Individuo 1 & Individuo 2 & Basural 1 \\
\hline Húmero & 41,1 (i); 41,16 (d) & 45 (d) & - \\
\hline Radio & 41,85 (i); 41,85 (d) & - & - \\
\hline Ulna & 42,65 (i) & - & - \\
\hline Fémur & 41,37 (d) & - & 42,52 (i, Til 1_n21) \\
\hline Tibia & 41,33 (i); 41,17 (d) & - & - \\
\hline
\end{tabular}

Húmero + radio 41,32 (i); 41,35 (d)

Fémur + tibia 41,35 (d) - $\quad$ -

\begin{tabular}{llll}
\hline Metacarpo II & 40,81 (i) & - & - \\
\hline
\end{tabular}

Metacarpo III 40,9 (i); 40,63 (d) - - -

Metacarpo IV 40,13 (i); 40,11 (d) - - -

\begin{tabular}{|c|c|}
\hline Metacarpo V & 40,56 (i); 40,33 (d) \\
\hline
\end{tabular}

\begin{tabular}{llll}
\hline Metatarso II $\quad 40(\mathrm{i}) ; 40,35$ (d) & - & - \\
\hline
\end{tabular}

Metatarso III $\quad 42,45$ (i); 42,37 (d) - -

\begin{tabular}{llll}
\hline Metatarso IV & $40,36(\mathrm{i}) ; 40,05$ (d) & - & - \\
\hline
\end{tabular}

Metatarso V $\quad 41,06$ (i); 41,98 (d)

Tabla 8. Estimaciones del índice de robustez de la diáfisis para Canis lupus familiaris de Til 1.

Referencias: "(i)" izquierdo, "d" derecho.

Estimates of the index of shaft robustness for Canis lupus familiaris from the Til 1 site. References: "(i)" left, "(d)" right.

\begin{tabular}{lccc}
\hline Elemento & Individuo 1 & Individuo 2 & Basural 1 \\
\hline Húmero & $8,01(\mathrm{~d}) ; 7,86(\mathrm{~d})$ & 7,93 (d) & - \\
\hline Radio & $7,48(\mathrm{i}) ; 7,43(\mathrm{~d})$ & - & - \\
\hline Fémur & 7,61 (d) & - & 7,41 (i, Til 1_n21) \\
\hline Tibia & $6,71(\mathrm{i}) ; 6,58(\mathrm{~d})$ & - & - \\
\hline
\end{tabular}

animales de Til 1 que alcanzaron la adultez tenían miembros de robustez mediana (Tabla 8) y una alzada que rondaba los 41-45 cm (Tabla 7), de manera que se corresponderían con tipos eumétricos (De Grossi Mazzorin y Tagliacozzo 2000).

Ninguno de los ejemplares de Til 1 exhibe características morfodentarias de los perros sin pelo americanos (p.ej., hipodoncia y falta de desarrollo de las cúspides dentarias, véase Kupczik et al. 2017; Vásquez et al. 2019). De esta manera, pese a la propuesta de Cabrera (1934) de la presencia de estos en Tilcara y a las numerosas observaciones

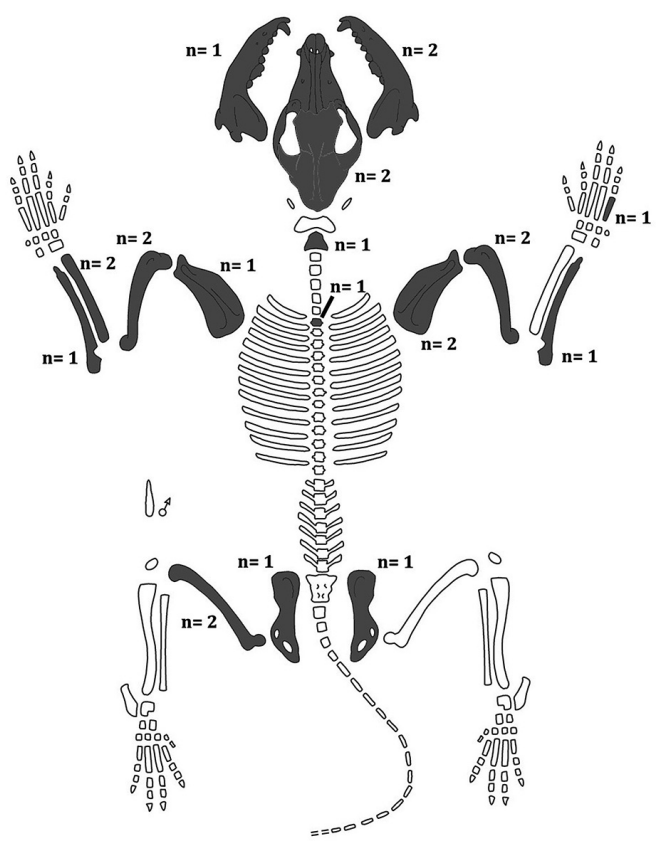

\section{Dientes aislados: C1 (d), c1 (i) y $\mathrm{m1}$ (d)}

Figura 5. Representación anatómica de la muestra de Canis lupus familiaris recuperada en el Basural 1 de Til 1. Figura muda tomada de ArcheoZoo.org (2013).

Anatomical representation of the Canis lupus familiaris sample recovered from Midden 1 of Til 1 site. Blank figure taken from ArcheoZoo.org (2013).

efectuadas por cronistas desde Mesoamérica hasta el Cono Sur (Allen 1920; Schwartz 1997; Valadez Azúa et al. 1998; Weiss 1970), hasta el momento en Argentina no existen registros zooarqueológicos de perros sin pelo.

\section{Posibles roles de los perros de Til 1}

Los perros pudieron cumplir múltiples roles dentro de las sociedades de las cuales formaban parte, incluso dentro del mismo sitio o periodo temporal (Hill 2013; Russell 2012). Es esperable que Til 1, al ser un asentamiento grande y ocupado durante todo el año, haya contribuido a mantener una población estable de perros aldeanos/callejeros y que una gran parte de estos subsistiesen de los desechos. De todos modos, esta última propuesta debe ser contrastada con el análisis tafonómico de los restos arqueofaunísticos recuperados, por ejemplo, en los basurales del sitio.

Dado que la población residente de Til 1 desarrolló tareas de producción artesanal especializada y que no se hallaron objetos relacionados con la producción 


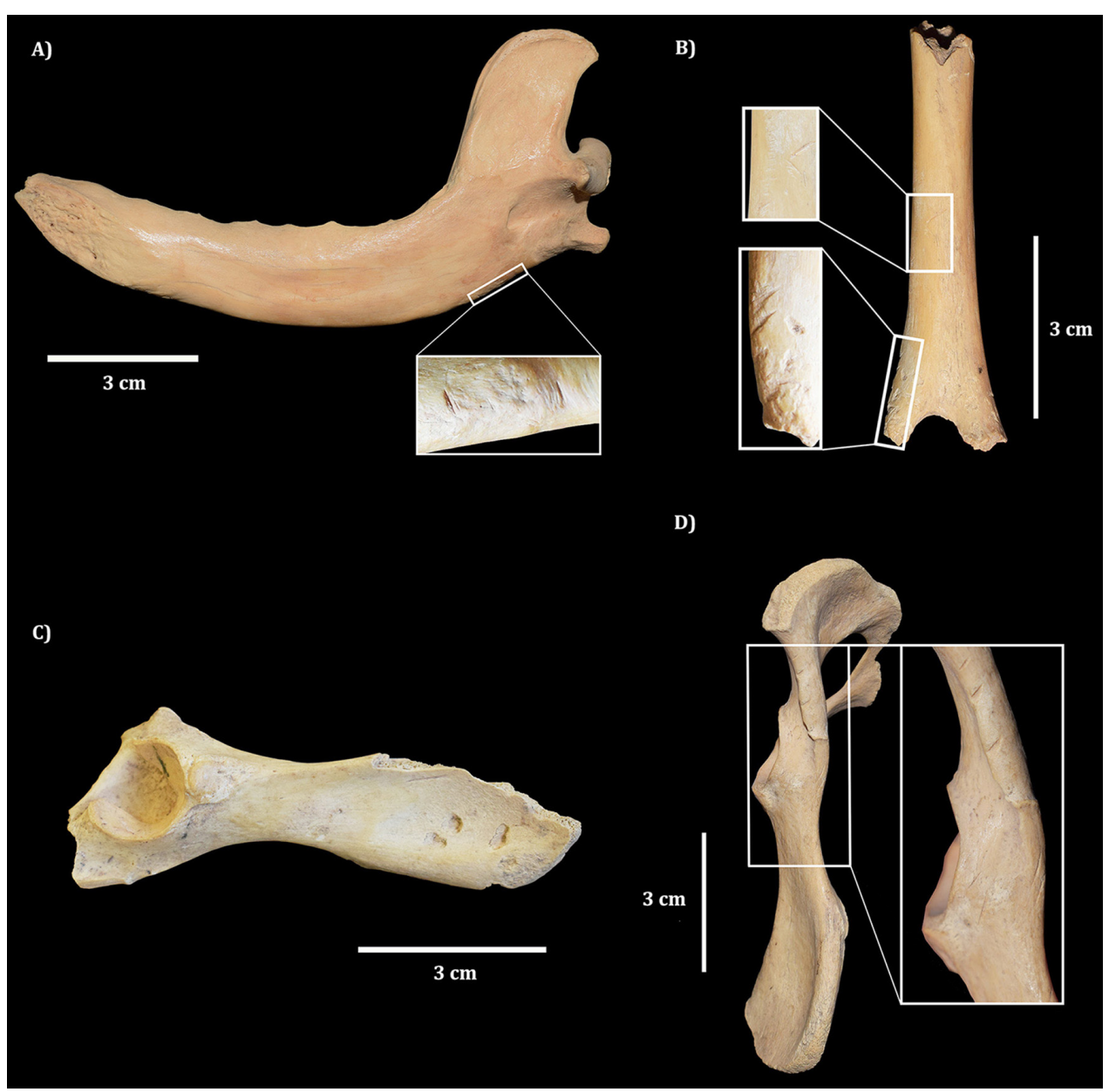

Figura 6. Especímenes del Basural 1 con evidencias de manipulación antrópica y de agentes tafonómicos naturales. (A) Hemimandíbula (Til 1_n3) con huellas de corte profundas sobre la base de la rama horizontal; (B) húmero (Til 1_n17) con huellas de corte en diáfisis medial y sobre la cresta supracondilar; (C) hemipelvis (Til 1_n19) con marcas de dientes de carnívoro en el íleon, y (D) hemipelvis (Til 1_n20) con múltiples huellas de corte profundas.

Specimens from Midden 1 with evidence of anthropic manipulation and natural taphonomic agents. (A) Hemimandible (Til 1_n3) with deep cut marks on the base of the horizontal ramus; (B) humerus (Til 1_n17) with cut marks in the medial shaft and the supracondylar ridge; (C) os coxae (Til 1_n19) with carnivorous teeth marks on the ileum and (D) os coxae (Til 1_n20) with multiple deep cut marks.

agrícola (Otero 2013), es posible que los perros del sitio no hayan estado asociados con funciones o labores específicas de trabajo (p.ej., como auxiliares en actividades de caza o de pastoreo). Por el contrario, debido a que los individuos 1 y 2 de la Acrópolis no presentan huellas de procesamiento antrópico y a que fueron recuperados en sectores de vivienda de miembros de la élite incaica y en talleres de producción de bienes suntuarios, parecen más compatibles con animales de compañía (mascota) y/o de prestigio, probablemente propiedad de algún miembro de la élite o de un artesano. Es esperable que en estos casos los perros hayan adquirido, en algunos aspectos, un estatus similar al de los humanos y, por lo tanto, un tratamiento después de la muerte también similar (p.ej., con ajuar funerario semejante y sepultado en el mismo espacio que las personas) (Hill 2013; Losey et al. 2011; Prates 2014).

Además de los indicadores que sugieren que algunos de los perros de la Acrópolis fueron animales de compañía y/o prestigio, también registramos huellas de origen antrópico en los restos recuperados en el Basural 1. Estas huellas tafonómicas indican un procesamiento integral de algunas carcasas, como 
Tabla 9. Evidencias de manipulación antrópica registradas en los especímenes de Canis lupus familiaris recuperados en el Basural 1. Evidence of anthropic manipulation recorded in Canis lupus familiaris specimens recovered from Midden 1.

\begin{tabular}{|c|c|c|c|}
\hline Elemento & Ubicación & Tipo de huella & Tipo de procesamiento/manipulación \\
\hline Hemimandíbula (Til 1_n3) & Rama horizontal (borde ventral) & Huellas de corte & Cuereo \\
\hline \multirow{4}{*}{ Escápula (Til 1_n9) } & $\begin{array}{l}\text { Fosa infraespinosa, supraespinosa y espina } \\
\text { escapular (cara lateral) }\end{array}$ & Huellas de corte & Descarne \\
\hline & Cuello (cara lateral) & Huellas de corte & Descarne y/o desarticulación \\
\hline & Borde caudal y craneal (cara medial) & Huellas de corte & Descarne \\
\hline & Fosa subescapular (cara medial) & Huellas de corte & Desarticulación \\
\hline \multirow[b]{2}{*}{ Húmero (Til 1_n11) } & Diáfisis (cara lateral y caudal) & Posibles marcas de dientes humanos & ¿Consumo? \\
\hline & $\begin{array}{l}\text { A lo largo de toda la diáfisis (principalmente } \\
\text { cara medial) }\end{array}$ & Huellas de corte y raspado & Descarne \\
\hline Húmero (Til 1_n12) & $\begin{array}{l}\text { A lo largo de toda la diáfisis (principalmente } \\
\text { cara medial) }\end{array}$ & Huellas de corte y raspado & Descarne \\
\hline Ulna (Til 1_n13) & Diáfisis distal (cara lateral) & Huellas de corte & Descarne y/o cuereo \\
\hline \multirow{2}{*}{ Fémur (Til 1_n14) } & Diáfisis medial (cara caudal) & Fractura en estado fresco & Posible consumo de médula ósea \\
\hline & Diáfisis medial (cara lateral) & Huellas de corte & Descarne \\
\hline \multirow{4}{*}{ Escápula (Til 1_n15) } & Espina escapular (cara lateral) & Huellas de corte & Descarne \\
\hline & $\begin{array}{l}\text { Posterior de fosa subescapular y facies serrata } \\
\text { (cara medial) }\end{array}$ & Huellas de corte & Desarticulación \\
\hline & Borde craneal (cara medial) & Huellas de corte & Descarne \\
\hline & Borde cavidad glenoidea (cara medial) & Huellas de corte & Desarticulación \\
\hline Húmero (Til 1_n16) & A lo largo de toda la diáfisis & Huellas de corte y raspado & Descarne \\
\hline \multirow{3}{*}{ Húmero (Til 1_n17) } & A lo largo de toda la diáfisis & Huellas de corte y raspado & Descarne \\
\hline & Diáfisis medial (cara labial) & Posibles marcas de dientes humanos & ¿Consumo? \\
\hline & Cresta supracondilar (cara lateral) & Huellas de corte & Desarticulación \\
\hline Ulna (Til 1_n18) & Diáfisis medial (cara medial) & Huellas de corte & Descarne y/o desarticulación \\
\hline \multirow{3}{*}{ Hemipelvis (Til 1_n19) } & Muesca isquiática mayor y espina alar (cara lateral) & Huellas de corte & Descarne \\
\hline & Cuerpo de íleon (cara medial) y espina alar & Huella de corte y fractura en estado fresco & Desarticulación \\
\hline & Isquion & Fractura en estado fresco & Desarticulación \\
\hline \multirow[b]{2}{*}{ Hemipelvis (Til 1_n20) } & $\begin{array}{l}\text { Alrededor de la fosa del acetábulo y del foramen } \\
\text { obturador (cara lateral) }\end{array}$ & Huellas de corte & Desarticulación y/o descarne \\
\hline & $\begin{array}{l}\text { Alrededor del foramen obturador, tuberosidad } \\
\text { isquiática, cuerpo de íleon y superficie auricular } \\
\text { (cara medial) }\end{array}$ & Huellas de corte & Desarticulación \\
\hline Fémur (Til 1_n21) & A lo largo de la diáfisis (cara craneal) & Huellas de corte y raspado & Descarne \\
\hline Vértebra toráxica (Til 1_n23) & Cuerpo vertebral & Huellas de corte & Desarticulación \\
\hline Radio (Til 1_n24) & Diáfisis distal (cara dorsal) & Huella de corte & Descarne y/o cuereo \\
\hline
\end{tabular}




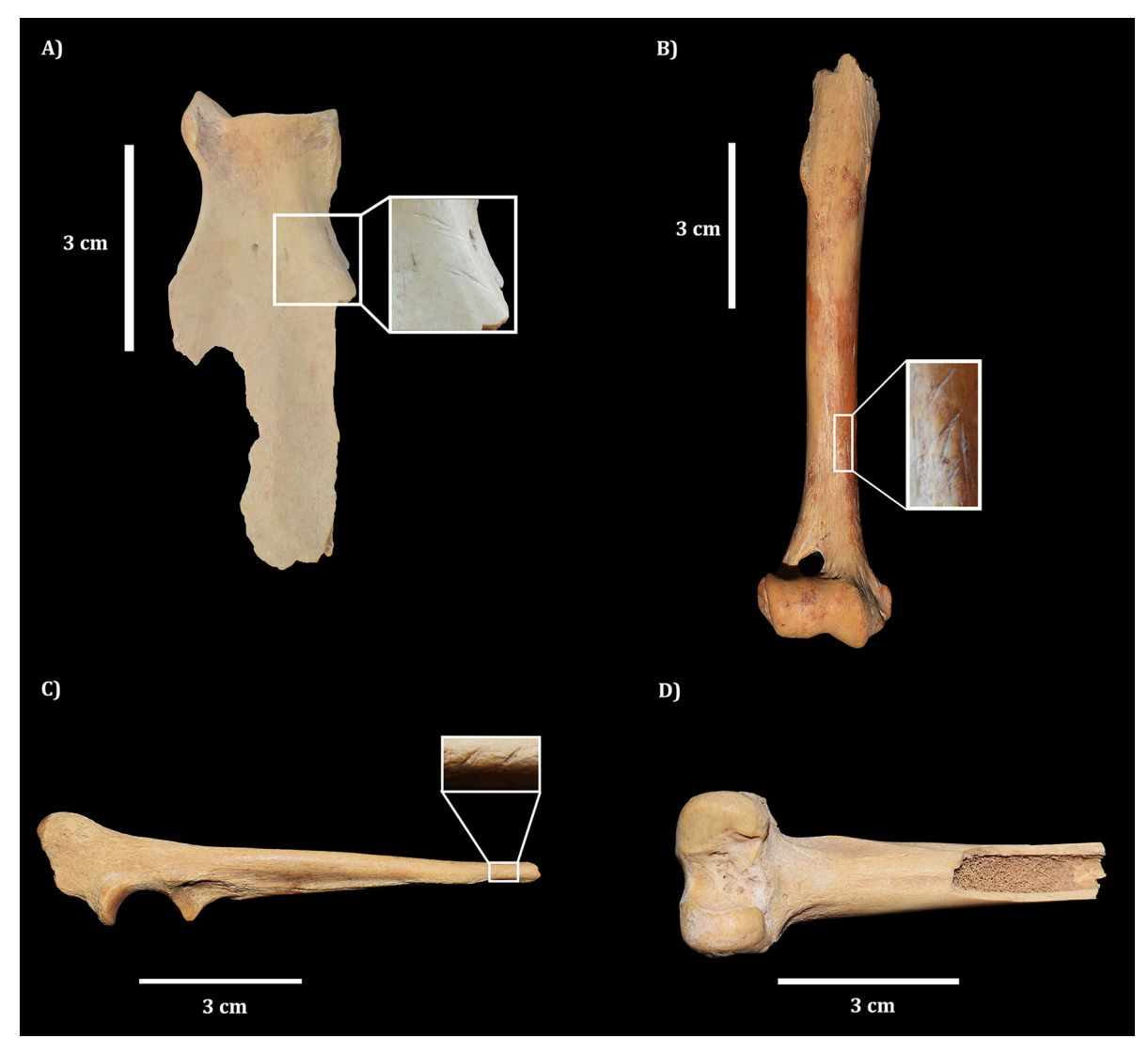

Figura 7. Especímenes del basural 1 con evidencias de manipulación antrópica. (A) Escápula (Til 1_n9) con huellas de corte en borde caudal; (B) húmero (Til 1_n12) con huellas de corte en diáfisis; (C) ulna (Til 1_n18) con huellas de corte en diáfisis y (D) fémur (Til 1_n14) con fractura producida en estado fresco.

Midden 1 specimens with evidence of anthropic manipulation. (A) Scapula (Til 1_n9) with cut marks on the caudal edge; (B) humerus (Til 1_n12) with cut marks on the diaphysis; (C) ulna (Til 1_n18) with cut marks on the diaphysis and (D) femur (Til 1_n14) with green fracture.

cuereo (implicando posiblemente la obtención del pelaje para la confección de vestimenta y/o diferentes objetos), desarticulación de la columna vertebral y de los miembros, descarne y probablemente obtención de médula ósea (Tabla 9). No hay evidencias de la utilización de huesos para materias primas. Si bien para el NOA el consumo de perros no fue discutido en profundidad y se encuentra en proceso de investigación, se planteó la posibilidad de su ocurrencia en cinco sitios. El primero fue Schuel (1930), quien propuso que los cráneos de los perros de Til 1 habrían sido consumidos dentro de un contexto ceremonial, basándose en las fracturas que presentaban en su parte posterior y por la ausencia de otros huesos del esqueleto. La imposibilidad de analizar los restos, debido a que se encuentran extraviados, y la poca información sobre el contexto de procedencia hacen difícil evaluar esta hipótesis.
Para el sitio Tastil (Quebrada del Toro, Salta), Zetti (1973) informó el hallazgo de restos de Canis lupus familiaris en basurales. Luego, Raffino et al. (1977) analizaron esos materiales y los del sitio Morohuasi, ubicado en la misma quebrada, y no encontraron evidencias de huellas de descarne que validen el consumo de perros. En el sitio La Huerta (Quebrada de Humahuaca, Jujuy), Madero (1993) se refirió a la presencia de huellas de corte en cánidos, aunque no pudo establecer si los restos correspondían a zorros o a perros. Por último, Rodríguez Loredo (1997-1998) observó posibles huellas antrópicas en distintos huesos de Canis lupus familiaris del sitio incaico Potrero-Chaquiago (Andalgalá, Catamarca).

Aunque la ingesta de perros ha sido documentada arqueológicamente (Chrószcz et al. 2015; Harcourt 1974; Martin et al. 2014; Murphy 2001; Russell 2012 , entre otros) y observada en la actualidad en 


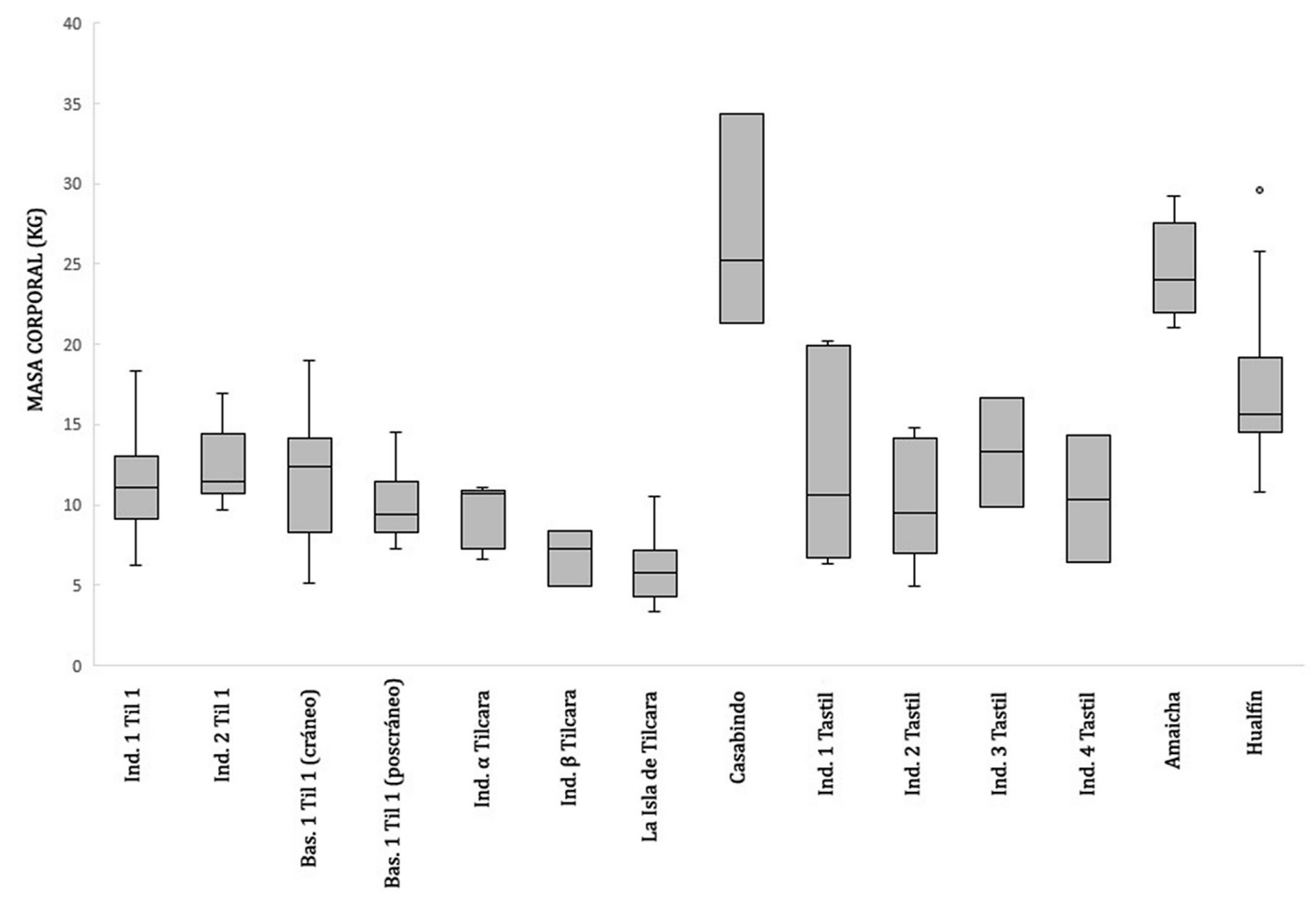

Figura 8. Estimaciones de masas corporales (kg) para los registros de Canis lupus familiaris arqueológicos del NOA. A partir de las medidas morfométricas tomadas por Von Ihering (1913), Cabrera (1934) y Zetti (1973), se las homologó con las relevadas por Von Den Driesch (1976) para realizar los cálculos. Referencias: Ind. 1 Til 1, Ind. 2 Ind. 1 Til 1, Bas. 1 Ind. 1 Til 1 (cráneo) y Bas. 1 Ind. 1 Til 1 (poscráneo) corresponden a los perros de Til 1 analizados en este trabajo; Ind. $\alpha$ Tilcara, Ind. $\beta$ Tilcara, Casabindo y Amaicha (Cabrera 1934); Hualfín (Von Ihering 1913; Cabrera 1934); La Isla de Tilcara (Belotti López de Medina 2017) y Tastil (Zetti 1973). Los especímenes del Basural 1 solo incluyen animales subadultos y adultos.

Body mass estimates $(\mathrm{kg})$ for the archaeological records of Canis lupus familiaris from NOA. Based on the morphometrical measurements taken by Von Ihering (1913), Cabrera (1934), and Zetti (1973), they were equated to those taken by Von Den Driesch (1976) to perform the calculations. References: Ind. 1 Til 1, Ind. 2 Til 1, Bas. 1 Til 1 (skull), and Bas. 1 Til 1 (postcranium) correspond to the Til 1 dogs analyzed in this article; Ind. $\alpha$ Tilcara, Ind. $\beta$ Tilcara, Casabindo and Amaicha (Cabrera 1934); Hualfin (Von Ihering 1913; Cabrera 1934); La Isla de Tilcara (Belotti López de Medina 2017); and Tastil (Zetti 1973). Midden 1 specimens comprise only subadult-adult animals.

distintos lugares del mundo (Garba et al. 2013; Podberscek 2009), no podría considerarse una práctica extendida ni predominante. Y como ocurre con el consumo de los carnívoros en general, el uso de los perros como alimento ha estado generalmente sujeto a complejos sistemas simbólicos de permisos y restricciones (Anthony y Brown 2017; Hayden 1990; Russell 2012; Schwartz 1997). Aunque las variables de este sistema son difíciles de aislar arqueológicamente, es interesante que, en Mesoamérica, donde no se disponía de animales domésticos que aporten considerable volumen cárnico, la práctica parece haber sido más frecuente que en el área Andina (Clutton-Brock y Hammond 1994; Russell 2012; Schwartz 1997; Valadez Azúa et al. 2013; Wing 1978), donde los camélidos fueron una fuente importante de proteínas. Esto implica que, si bien el valor nutritivo de los perros, en algunos casos, pudo determinar o potenciar la costumbre de consumirlos, no parece ser una explicación suficiente para el NOA.

Finalmente, si se asume que en el NOA la expansión del Estado inca se produjo aproximadamente entre 1370 y 1400 años DC (Marsh 2016; Williams 2000), y no en la fecha de 1420-1430 años DC establecida en la periodización de la Quebrada de Humahuaca (Nielsen 2001, 2007), la manipulación antrópica identificada en el húmero fechado del Basural 1, con 1383-1424 años cal. DC, implicaría una transgresión de las normas del Imperio, que prohibían el consumo 
de perros en los territorios bajo su dominio (Schwartz 1997:73-76; Weiss 1970:38). Esto sugiere como más probable que el consumo de perros debió producirse en un escenario de ausencia de dominio efectivo del Imperio sobre algunas esferas de la vida cotidiana de la población local durante las primeras etapas expansivas y/o de resistencia étnica de los grupos como método para reforzar sus prácticas ancestrales (véase discusión para Til 1 en Scaro y Otero 2019).

\section{Conclusiones}

El estudio de un número mínimo de cinco individuos inéditos de Canis lupus familiaris de Til 1 (Quebrada de Humahuaca, Provincia de Jujuy), que se suman a los registros previos (Cabrera 1934; Rusconi 2015 [1934]; Schuel 1930), lo convierten en el sitio prehispánico del Cono Sur con mayor cantidad de individuos publicados. Esta abundancia puede estar relacionada con las características del sitio (p.ej. de gran tamaño y ocupado durante todo el año), que habrían generado las condiciones propicias para que los perros subsistan de los desechos. En este trabajo se presentó el primer fechado-taxón del NOA, que ubica a la especie a fines del periodo de Desarrollos Regionales o inicios del periodo Inca, y ratifica lo planteado previamente sobre evidencias cronológicas contextuales. Estos hallazgos refuerzan el patrón de abundancia arqueológica de los perros en Argentina (Prates et al. 2010a, 2010b), con numerosos registros en el NOA, en contextos de sociedades sedentarias con cierta complejidad en la organización política y social y una economía basada en la agricultura y la ganadería de camélidos.

La reconstrucción morfológica señala que se trató de individuos de tamaño mediano pequeño a pequeño, de robustez mediana. Estarían presentes animales con cráneos de tipo mesocéfalo (bien proporcionados entre su largo y ancho) y posiblemente de hocico corto, pero sin evidencias de perros sin pelo americanos. Los perros de pequeña envergadura parecen haber sido los más abundantes en el NOA (Belotti López de Medina 2012; Cabrera 1934; Raffino et al. 1977; Zetti 1973).

En cuanto a las funciones sociales, los perros hallados en la Acrópolis habrían funcionado como animales de compañía y/o de prestigio, y los del Basural 1 como recursos para el consumo de su carne y tal vez para el de uso de sus pieles. Aunque estas son las primeras evidencias indiscutibles del consumo de perros en el NOA en tiempos prehispánicos, no es posible determinar en qué medida esto formó parte de actividades exclusivamente de subsistencia o con algún componente ritual.

Agradecimientos: Este trabajo se desarrolló en el marco de una Beca Interna Doctoral del CONICET del primer autor. Esta investigación fue financiada por los proyectos ANPCyT (PICT-2015-3645, dirigido por Luciano Prates, PICT-2015-966 y PICT-2016-3151 dirigidos por Francisco Juan Prevosti) y CONICET (PIP244-15, dirigido por Luciano Prates). Queremos agradecer especialmente al Área de Antropología Biológica y al Archivo del Museo Etnográfico J.B. Ambrosetti de Buenos Aires, a la Dra. Mónica Berón y al Lic. Iván Díaz. A tres evaluadores anónimos, que ayudaron a mejorar este trabajo. Para el mapa de la Figura 1 utilizamos datos vectoriales y ráster en QGIS obtenidos de Natural Earth Data (https://www.naturalearthdata.com) y del Instituto Geográfico Nacional de la República Argentina (http:// www.ign.gob.ar).

\section{Referencias Citadas}

Acosta, A., D. Loponte y C. García Esponda 2011. Primer registro de perro prehispánico (Canis familiaris) entre los grupos cazadores recolectores del humedal de Paraná inferior (Argentina). Antipoda. Revista de Antropología y Arqueología 294 (13):175-199.

Allen, G.M. 1920. Dogs of the american aborigines. Bulletin of the Museum of Comparative Zoology 63:431-517.

Anthony, D.W. y D.R. Brown 2017. The dogs of war: A bronze age initiation ritual in the russian steppes. Journal of Anthropological Archaeology 48:134-148.

Belotti López de Medina, C.R. 2012. En compañía de los muertos. Ofrendas de animales en los cementerios de La Isla (Tilcara, Jujuy). Intersecciones en Antropología 13:345-357.
Belotti López de Medina, C.R. 2017. Morphometry of a prehispanic dog from northwestern Argentina (AD 800-1200). International Journal of Osteoarchaeology 27 (4):700-706.

Berta, A. 1989. Quaternary evolution and biogeography of the large south american canidae (mammalia: carnivora). University of California Publications in Geological Science 132:1-149.

Binford, L. 1981. Bones. Ancient Men and Modern Myths. Academic Press, New York.

Brassard, C. y C. Callou 2020. Sex determination of archaeological dogs using the skull: Evaluation of morphological and metric traits on various modern breeds. Journal of Archaeological Science: Reports 31:102294. 
Brothwell, D., A. Malaga y R. Burleigh 1979. Studies on amerindian dogs, 2: Variation in early peruvian dogs. Journal of Archaeological Science 6 (2):139-161.

Cabrera, A.L. 1934. Los perros domésticos de los indígenas del territorio argentino. Actas y Trabajos del XXV Congreso Internacional de Americanistas Vol. 1, pp. 81-93. Coni, Buenos Aires.

Casanova, E., H. Difrieri, N. Pelissero y J. Balbuena 1976. Un corte estratigráfico en el Pucará de Tilcara. Actas y Memorias del IV Congreso Nacional de Arqueología. Revista del Museo de Historia Natural T.III (1/4):21-30.

Castro, J.C., M. Bonomo, L. González Venanzi y S. Cornero 2020. Perros indígenas en el noreste argentino. Latin American Antiquity 31 (4):853-870.

Chrószcz, A., M. Janeczek, Z. Bielichová, T. Gralak y V. Onar 2015. Cynophagia in the Púchov (Celtic) culture settlement at Liptovská Mara, northern Slovakia. International Journal of Osteoarchaeology 25 (4):528-538.

Clark, K.M. 1995. The later prehistoric and protohistoric dog: the emergence of canine diversity. Archaeozoologia 7 (2):9-32.

Clutton-Brock, J. y N. Hammond 1994. Hot dogs: comestible canids in preclassic Maya culture at Cuello, Belize. Journal of Archaeological Science 21 (6):819-826.

De Grossi Mazzorin, J. y A. Tagliacozzo 2000. Morphological and osteological changes in the dog from the neolithic to the roman period in Italy. En Dogs Through Time: An Archaeological Perspective, editado por S.J. Crockford, pp. 141-161. Archaeopress, Oxford.

Evans, H.E. y A. de Lahunta 2013. Miller's Anatomy of the Dog. Cuarta edición, Saunders, Elsevier, St. Louis.

Fernández-Jalvo, Y. y P. Andrews 2016. Atlas of Taphonomic Identifications: 1001 + Images of Fossil and Recent Mammal Bone Modification. Springer, Dordrecht.

Gallardo, G. 1964-1965. Perros americanos precolombinos. Cuadernos del Instituto Nacional de Antropología y Pensamiento Latinoamericano 5:31-69.

Garba, A., A.A. Dzikwi, P.A. Okewole, B.B. Chitunya-Wilson, A.B. Tirmidhi, H.M. Kazeem y J.U. Umoh 2013. Evaluation of dog slaugther and consumption practices related to the control of rabies in Nigeria. Journal of Experimental Biology and Agricultural Sciences 1:125-130.

González, A.R. 1982. Las provincias inca del antiguo Tucumán. Revista del Museo Nacional 46:317-380.

Greco, C. y C. Otero 2016. The chronology of settlements with pre-inca and inca occupation superimposed: the case of Pucará de Tilcara (Humahuaca Gorge, Argentina). Archaeometry 58 (5):848-862.

Harcourt, R.A. 1974. The dog in prehistoric and early historic Britain. Journal of Archaeological Science 1 (2):151-175.

Hayden, B. 1990. Nimrods, piscators, pluckers, and planters: the emergence of food production. Journal of Anthropological Archaeology 9:31-69.

Hildebrand, M. 1954. Comparative morphology of the body skeleton in recent canidae. University of California Publication in Zoology 52:399-496.
Hill, E. 2013. Archaeology and animal persons. Toward a prehistory of human-animal relations. Environment and Society 4 (1):117-136.

Hogg, A.G., Q. Hua, P.G. Blackwell, M. Niu, C.E. Buck, T.P. Guilderson, T.J. Heaton, J.G. Palmer, P.J. Reimer y R.W. Reimer 2013. SHCal13 southern hemisphere calibration, 0-50,000 years cal BP. Radiocarbon 55 (4):1889-1903.

Horard-Herbin, M.P. 2000. Dog management and use in the late iron age: evidence from the gallic site of Levroux (France). En Dogs Through Time: An Archaeological Perspective, editado por S.J. Crockford, pp. 115-121. Archaeopress, Oxford.

Kupczik, K., A. Kagan, S. Brauer y M.S. Fischer 2017. The dental phenotype of hairless dogs with FOXI3 haploinsufficiency. Scientific Reports 7 (1):1-8.

Lawrence, B. 1967. Early domestic dogs. Zeitschrift für Säugetierkunde 32:44-59.

Legendre, S. y C. Roth 1988. Correlation of carnassial tooth size and body weight in recent carnivores (mammalia). Historical Biology 1 (1):85-98.

López Geronazzo, L.N., C. Otero, A. Álvarez, M.D. Ercoli y N. Cortés-Delgado 2019. Identification of guinea pig remains in the Pucará de Tilcara (Jujuy, Argentina): Evidence in favour of the presence of the andean breed in the Quebrada de Humahuaca. International Journal of Osteoarchaeology 29 (6):934-946.

López Mazz, J.M., F. Moreno, R. Bracco y R. González 2018. Perros prehistóricos en el este de Uruguay: contextos e implicaciones culturales. Latin American Antiquity 29 (1):64-78.

Loponte, D. y A. Acosta 2016. Nuevos registros prehispánicos de Canis familiaris (carnivora, canidae) en la cuenca del Paraná, Argentina. Mastozoología Neotropical 23 (2):431-454.

Losey, R.J., V.I. Bazaliiskii, S. Garvie-Lok, M. Germonpré, J.A. Leonard, A.L. Allen, M.A. Katzenberg y M.V. Sablin 2011. Canids as persons: Early neolithic dog and wolf burials, Cis-Baikal, Siberia. Journal of Anthropological Archaeology 30 (2):174-189.

Losey, R.J., B. Osipov, R. Sivakumaran, T. Nomokonova, E.V Kovychev y N.G Diatchina 2015. Estimating body mass in dogs and wolves using cranial and mandibular dimensions: application to siberian canids. International Journal of Osteoarchaeology 25 (6):946-959.

Losey, R.J., K. McLachlin, T. Nomokonova, K. Latham, L. Harrington 2017. Body mass estimates in dogs and north american gray wolves using limb elements dimensions. International Journal of Osteoarchaeology 27 (2):180-191.

Lyman, L.R. 2008. Quantitative Paleozoology. Cambridge University Press, New York City.

Madero, C. 1993. Explotación faunística, tafonomía y economía en la Quebrada de Humahuaca antes y después de los Yupanki. En lnka: Arqueología, Historia y Urbanismo del Altiplano Andino, editado por R. Raffino, pp. 141-168. Editorial Corregidor, Buenos Aires.

Marsh, E.J. 2016. ¿Cuándo llegaron los incas a Mendoza? Una reevaluación de los fechados radiométricos mediante un modelo bayesiano. Actas del XIX Congreso Nacional de Arqueología Argentina. pp. 1906-1913. Facultad de Ciencias 
Naturales e Instituto Miguel Lillo de la Universidad Nacional de Tucumán, Tucumán.

Martin, P., P. Saladié, J. Nadal y J.M. Vergès 2014. Butchered and consumed: small carnivores from the holocene levels of El Mirador Cave (Sierra de Atapuerca, Burgos, Spain). Quaternary International 353:153-169.

Milheira, R.G., D.M. Loponte, C. García Esponda, A. Acosta y P. Ulguim 2017. The first record of a pre-columbian domestic $\operatorname{dog}$ (Canis lupus familiaris) in Brazil. International Journal of Osteoarchaeology 27 (3):488-494.

Morey, D.F. 1992. Size, shape and development in the evolution of the domestic dog. Journal of Archaeological Science 19 (2):181-204.

Murphy, E.M. 2001. Medieval and post-medieval butchered dogs from Carrickfergus, Co. Antrim, northern Ireland. Environmental Archaeology 6 (1):13-22.

Nielsen, A.E. 2001. Evolución social en la Quebrada de Humahuaca (AD $700 \pm 1536$ ). En Historia Argentina Prehispánica, editado por E.E. Berberian y A.E. Nielsen, pp. 171-264. Editorial Brujas, Córdoba.

Nielsen, A.E. 2007. El período de desarrollos regionales en la Quebrada de Humahuaca: aspectos cronológicos. En Sociedades Precolombinas Surandinas: Temporalidad, Interacción y Dinámica Cultural del NOA en el Ámbito de los Andes Centro-Sur, editado por V.I. Williams, B.N. Ventura, A.B. M. Callegari y H.D. Yacobaccio, pp. 235-247. Taller Internacional de Arqueología del NOA y Andes Centro Sur, Buenos Aires.

Otero, C. 2013. Producción, Usos y Circulación de Bienes en el Pucará de Tilcara (Quebrada de Humahuaca, Jujuy). Tesis doctoral, Facultad de Filosofía y Letras, Universidad de Buenos Aires, Buenos Aires.

Otero, C. y M.N. Tarragó 2017. Reconstructing inca socioeconomic organization through biography analyses of residential houses and workshops of Pucara de Tilcara (Quebrada de Humahuaca, Argentine). Journal of Anthropology and Archaeology 5 (1):55-72.

Otero, C., M.B. Cremonte y P.A. Ochoa 2018. La construcción del poder incaico en la Quebrada de Humahuaca (Jujuy, Argentina). En Interpretando Huellas: Arqueología, Etnohistoria y Etnografía de los Andes y sus Tierras Bajas, editado por M.A. Muñoz, pp. 227-245. Grupo Editorial Kipus, Cochabamba.

Pelissero, N., C. Forgione y R. Alancay 1997. El Pucará de Tilcara. Colección Mankacén, Buenos Aires.

Podberscek, A.L. 2009. Good to pet and eat: the keeping and consuming of dogs and cats in South Korea. Journal of Social Issues 65 (3):615-632.

Prates, L. 2014. Crossing the boundary between humans and animals: the extinct fox Dusicyon avus from a hunter-gatherer mortuary context in Patagonia (Argentina). Antiquity 88 (342):1201-1212.

Prates, L., M. Berón y F.J. Prevosti 2010a. Los perros prehispánicos del cono sur. Tendencias y nuevos registros. En Mamül Mapu: Pasado y Presente desde la Arqueología Pampeana, editado por M. Berón, L. Luna, M. Bonomo, C. Montalvo, C. Aranda y M. Carrera Aizpitarte, pp. 215-228. Editorial Libros del Espinillo, Ayacucho.

Prates, L., F.J. Prevosti y M. Berón 2010b. First records of prehispanic dogs in southern south america (Pampa-Patagonia, Argentina). Current Anthropology 51 (2):273-280.
Prevosti, F.J. 2006. Grandes Cánidos (Carnívora, Canidae) del Cuaternario de la República Argentina: Sistemática, Filogenia, Bioestratigrafía y Paleoecología. Tesis doctoral, Facultad de Ciencias Naturales y Museo, Universidad Nacional de la Plata, Provincia de Buenos Aires.

Raffino, R.A, E.P. Tonni y A.L. Cione 1977. Recursos alimentarios y economía en la región de la Quebrada del Toro, provincia de Salta, Argentina. Relaciones de la Sociedad Argentina de Antropología 11:9-30.

Rodríguez Loredo, C. 1997-1998. Estudio arqueozoológico del sitio inca Potrero Chaquiago, barrios La Solana y Retambay, Andalgalá, pcia. de Catamarca (Argentina). Relaciones de la Sociedad Argentina de Antropología 22-23:203-245.

Romero, A.J., J.C. Diez y P. Saladié 2016. Mammal bone surface alteration during human consumption: an experimental approach. Journal of Archaeological Science 8:82-89.

Rusconi, C. 2015 [1934]. Nuevos restos de vertebrados vivientes y extinguidos de los túmulos prehispánicos de Santiago del Estero. En La Civilización Chaco-Santiagueña y sus Correlaciones con las del Viejo y Nuevo Mundo, editado por E.R. Wagner y D.L. Wagner, pp. 248-257. Fundación Cultural Santiago del Estero, Santiago del Estero.

Russell, N. 2012. Social Zooarchaeology. Humans and Animals in Prehistory. Cambridge University Press, New York City.

Saladié, P., A. Rodriguez-Hidalgo, C. Diez, P. Martín-Rodríguez y E. Carbonell 2013. Range of bone modifications by human chewing. Journal of Archaeological Science 40:380-397.

Scaro, A. y C. Otero 2019. Inca mortuary practices. Material accounts of death in Quebrada de Humahuaca at the time of the empire. Global Journal of Archaeology and Anthropology 9 (1):1-12.

Schuel, K. 1930. Ruinas de las poblaciones indígenas de la provincia de Jujuy. Actas de la V Reunión Sociedad Argentina de Patología Regional del Norte Argentino Vol. 2, pp.1430-1451. Imprenta de la Universidad, Buenos Aires.

Schwartz, M. 1997. A History of Dogs in the Early Americas. New Have, CT, Yale University Press, New Haven.

Snyder, L.M. 1995. Assessing the Role of the Domestic Dog as a Native American Food Resource in the Middle Missouri Subarea A.D. 1000-1840. Tesis doctoral, Department of Anthropology, University of Tennessee, Tennessee.

Stuiver, M. y P.J. Reimer 1993. Extended ${ }^{14} \mathrm{C}$ database and revised CALIB $3.0{ }^{14} \mathrm{C}$ calibration program. Radiocarbon 35 (1):215-230

Sutton, L.K., J.H. Byrd y J.W. Brooks 2018. Age determination in dogs and cats. En Veterinary Forensic Pathology, editado por J.W. Brooks, pp. 151-163. Springer, Cham.

Tarragó, M.N. 2000. Chacras y pukara. Desarrollos sociales tardíos. En Los Pueblos Originarios y la Conquista, editado por M.N. Tarragó, pp. 257-300. Sudamericana, Buenos Aires.

Tarragó, M.N. y M.E. Albeck 1997. Fechados radiocarbónicos para el sector medio de la Quebrada de Humahuaca. Avances en Arqueología 3:101-129.

Tedford, R., B.E. Taylor y X. Wang 1995. Phylogeny of the caninae (carnivore: canidae): The living taxa. American Museum Novitates 3146:1-37. 
Val, A. y J.B. Mallye 2011. Small carnivore skinning by professionals: skeletal modifications and implications for the european upper palaeolithic. Journal of Taphonomy 9 (4):221-243.

Valadez Azúa, R., A. Blanco y B. Rodríguez Galicia 1998. Restos arqueozoológicos de xoloitzcuintles (1994-1998). Asociación Mexicana de Médicos Veterinarios Especialistas en Pequeñas Especies (AMMVEPE) 9 (6):181-190.

Valadez Azúa, R., A. Blanco Padilla, B. Rodríguez Galicia y G. Pérez Roldán 2013. The dog in the Mexican archaeozoological record. En The Archaeology of Mesoamerican Animals, editado por C.M. Götz y K.F. Emery, pp. 557-582. Archaeobiology 1, Lockwood Press, Atlanta, Georgia.

Vásquez, V.F., T.E. Rosales, G. Dorado, P. Allemant y F. Darleguy 2019. El gen FOXI3 y sus repercusiones zooarqueológicas en el "perro sin pelo del Perú" (Canis lupus familiaris) - Revisión. Archaeobios 1 (13):87-102.

Vásquez, V.F., T.E. Rosales, C. Gálvez y G. Dorado 2016. El origen del perro (Canis lupus familiaris) sin pelo peruano (PSPP): pruebas arqueológicas, zooarqueológicas y genéticas - Revisión. Archaeobios 1 (10):80-102.

Von Den Driesch, A. 1976. A Guide to the Measurement of Animal Bones from Archaeological Sites. Harvard University Press, Cambridge.

Von Ihering, H. 1913. Le chien domestique des calchaquís. Revista del Museo de La Plata 20:101-106.
Weiss, P. 1970. El perro peruano sin pelo (perro chino, viringo, ccala o ccalato). Acta Herediana 3 (1):33-54.

Williams, V.I. 2000. El imperio inka en la provincia de Catamarca. Intersecciones en Antropología 1 (1):55-78.

Williams, V.I. 2004. Poder estatal y cultura material en el kollasuyu. Boletín de Arqueología PUCP 8:209-245.

Wing, E.S. 1978. Use of dogs for food: an adaptation to the coastal environment. En Prehistoric Coastal Adaptation: The Economy and Ecology of Maritime Middle America, editado por B.L. Stark y B. Voorhies, pp. 29-41. Academic Press, New York City.

Zaburlín, M.A. 2009. Historia de ocupación del Pucará de Tilcara (Jujuy, Argentina). Intersecciones en Antropología 10:89-103.

Zaburlín, M.A. y C. Otero 2014. Un manuscrito olvidado de J.B. Ambrosetti: "Exploraciones arqueológicas en la antigua ciudad del Pukará de Tilcara". En Investigaciones del Instituto Interdisciplinario Tilcara, editado por M.E. Aparicio, A. Benedetti, L. Bugallo, L.M. Mamaní, P. Mercolli, M. Montenegro, C. Otero, L. Haydée Paredes, C. Rivet, J. Tomasi, M. Weinberg y M.A. Zaburlín, pp. 161-220. Editorial de la Facultad de Filosofía y Letras de la Universidad de Buenos Aires, Buenos Aires.

Zetti, J. 1973. Paleoetnozoología del Tastil. En Tastil, Una ciudad Preincaica Argentina, editado por E.M. Cigliano, pp. 565-578. Ediciones Cabargon, Buenos Aires.

\section{Nota}

1 Se desestimaron los fechados realizados por Pelissero et al. (1997) por presentar diversos problemas metodológicos (véase Otero 2013:170-171). 
